\title{
Review of high-speed imaging with lab-based x-ray computed tomography
}

\author{
E A Zwanenburg*(D), M A Williams $\mathbb{D}$ and J M Warnett \\ WMG, University of Warwick, Coventry, United Kingdom \\ E-mail: Evelien.Zwanenburg@warwick.ac.uk
}

Received 21 April 2021, revised 22 October 2021

Accepted for publication 1 November 2021

Published 19 November 2021

\begin{abstract}
$\mathrm{X}$-ray computed tomography $(\mathrm{CT})$ is frequently used for non-destructive testing with many applications in a wide range of scientific research areas. The difference in imaging speeds between synchrotron and lab-based scanning has reduced as the capabilities of commercially available CT systems have improved, but there is still a need for faster lab-based CT both in industry and academia. In industry high-speed CT is desirable for inline high-throughput CT at a higher resolution than currently possible which would save both time and money. In academia it would allow for the imaging of faster phenomena, particularly dynamic in-situ testing, in a lab-based setting that is more accessible than synchrotron facilities. This review will specifically highlight what steps can be taken by general users to optimise scan speed with current equipment and the challenges to still overcome. A critical evaluation of acquisition parameters across recent high-speed studies by commercial machine users is presented, indicating some areas that could benefit from the methodology described. The greatest impacts can be achieved by maximising spot size without notably increasing unsharpness, and using a lower number of projections than suggested by the Nyquist criterion where the anecdotal evidence presented suggests usable results are still achievable.
\end{abstract}

Supplementary material for this article is available online

Keywords: x-ray microscopy, computed tomography, high-speed XCT, micro CT, in-situ, dynamic CT

(Some figures may appear in colour only in the online journal)

\section{Introduction}

Originally a medical diagnostic tool, $\mathrm{x}$-ray computed tomography (CT) has become common in non-destructive testing (NDT) in the last few decades. The imaging process allows

Author to whom any correspondence should be addressed.

Original Content from this work may be used under the terms of the Creative Commons Attribution 4.0 licence. Any further distribution of this work must maintain attribution to the author(s) and the title of the work, journal citation and DOI. internal inspection of complex objects without dismantling or damaging them, leading to prominence in a number of diverse applications from materials science and industry to forensics and museums. For example, in forensics it has been revolutionary in tool mark analysis by scanning a piece of bone to identify how the injury was sustained [1-3]. This type of analysis has even been presented as part of a live court case [4]. The geosciences have many applications from the study of porosity in rocks and grain analysis, to fluid flows therein [5-10]. Aside from gaining more information on an object it also allows for the digital preservation of specimens in natural history collections that can degrade over time [11-15]. 
Materials scientists are particularly interested in the response to loading, and use CT to study anything from damage and crack characterisation to fibre analysis in polymer composites [16-20]. Within life sciences traditional 2D microscopy has now significantly evolved thanks to CT imaging [21]. CT is not only a tool for academic research but it is also essential for industrial innovation particularly within manufacturing. Additive manufacturing (AM) is praised for its ability to directly print complex geometries of which CT is the only way to evaluate against the desired specifications as it provides internal visibility in any direction without consequence [22-24]. This is in contrast to other NDT methods such as optical inspection which provides purely external information, or ultrasonic testing that is less versatile in terms of the objects and requires preparation of the surface of the object. Measuring internal and external features to inspect dimensions and geometries of objects is critical in design for function [25], with research continuing to optimise repeatability and reproducibility as a metrological system [26, 27].

Although it is now possible to achieve scan acquisition times to the order of (tens of) minutes in lab-based facilities dependent on the sample, faster acquisition is still desired. The EPSRC Tomography Roadmap (2018) confirms this as it states that ' $39 \%$ of respondents indicated that speed was something that was hindering their research' and 'time resolved imaging was a future need of the community' [28]. This escalating interest can also be observed in the number of publications involving faster scanning applications in the last ten years as seen in figure 1 . Due to the limitation of lab-based hardware, traditionally faster scanning was largely performed at a synchrotron. It is clear that over the last 10 years this trend has changed.

Synchrotron CT scanning has a big advantage: the high intensity monochromatic beams allow for acquisition times of the order of seconds [29]. For sub-second acquisition times (potentially at sub-micron voxel sizes) a synchrotron source is still required, but lab-based machines have continually improved hardware with faster speeds at greater resolutions making them a viable alternative in many cases $[17,18,27]$. This is in addition to the much greater availability of lab-based CT facilities [16]. Exposure times have notably come down: in the nineties a high resolution scan would take days [30] whereas now exposure times as low as $20 \mathrm{~ms}$ are possible [29] enabling viable scan times of under a minute. More on hardware improvements and limitations for lab-based faster scanning can be found in Bultreys et al [31].

X-ray CT (XCT) standards such as BSI BS EN ISO 15708 [32] and VDI/VDE 2630 [33] focus on parameter control to optimise image quality for the most accurate and repeatable measurements. Different studies have been carried out on standardization from inter-laboratory comparisons [34, 35], to test objects for calibration and accuracy testing [36-39]. Multiple assessments highlight the need to provide better procedures by international standards: for example an international comparison performed by Carmignato [40] showed traceability of dimensional measurements is still problematic even for expert users. Moroni and Petrò [41] highlight the need for more procedures as they conclude that 'even expert operators have shown not to be able to choose the correct parameters to obtain the estimates of the performance parameters'. Also du Plessis et al [42] note that although micro-CT is a good inspection tool for metal AM, greater standardization and development of protocols is required to be accepted as the de facto assessment tool. While this maturation is ongoing, there is also a clear need for best practice in high-speed scanning detailing how to find the optimal balance between image quality and scanning speed. To find this balance image quality needs quantification but currently there is no standard holistic metric in XCT [43] and should be decided on a fit-for-purpose basis. Du Plessis et al [44] has proposed a metric standard that evaluates the image quality as a tool for quality control but it is limited to single material objects.

While sample dependent, and without loss of generality, a typical XCT acquisition can take upwards of an hour. The term high-speed is not explicitly defined for XCT but arguably it is when the parameters are adjusted beyond a standard protocol such that it is faster than it would normally be, and therefore typically (significantly) faster than an hour. To complete a scan as fast as possible, needs must be balanced against the application, where the resulting data of high-speed scans whether they are a minute or 10 min must be of sufficient quality to observe and measure what is required. The majority of articles on high-speed CT focuses on the application itself, mainly in-situ experiments, and not how they achieved the shortest acquisition time required. Often they provide their choice of scan parameters but fail to explain the rationale behind them and found this balance which is often the limiting factor.

The aim of this review is to highlight what trade offs can be made in the acquisition parameters for speed, analyse how labbased high-speed CT is currently being performed to identify any further anecdotal evidence, and where improvements can be made for even faster acquisition times. To begin different applications of fast scanning are discussed, followed by an explanation of the acquisition parameters and how they impact the acquisition time. Previously used parameters in faster CT scanning are interrogated for optimality, concluding with recommendations resulting from this review. This will give general users a view of current fast scanning practices and how they themselves can move forward in high-speed scanning, and expert users insights as to how they can improve their acquisition times.

\section{Applications of high-speed lab-based CT}

Faster scanning in the lab is desirable as it enables the imaging and measurement of dynamic phenomena which is predominantly only possible at central synchrotron facilities. Alternatively it allows for high-throughput, which is of interest to manufacturing lines where imaging would ideally occur at the rate of production and for identification of quality issues on individual parts in real-time. This section will first discuss developments in high-throughput CT and then highlight different applications in academia that will benefit from faster scanning. 


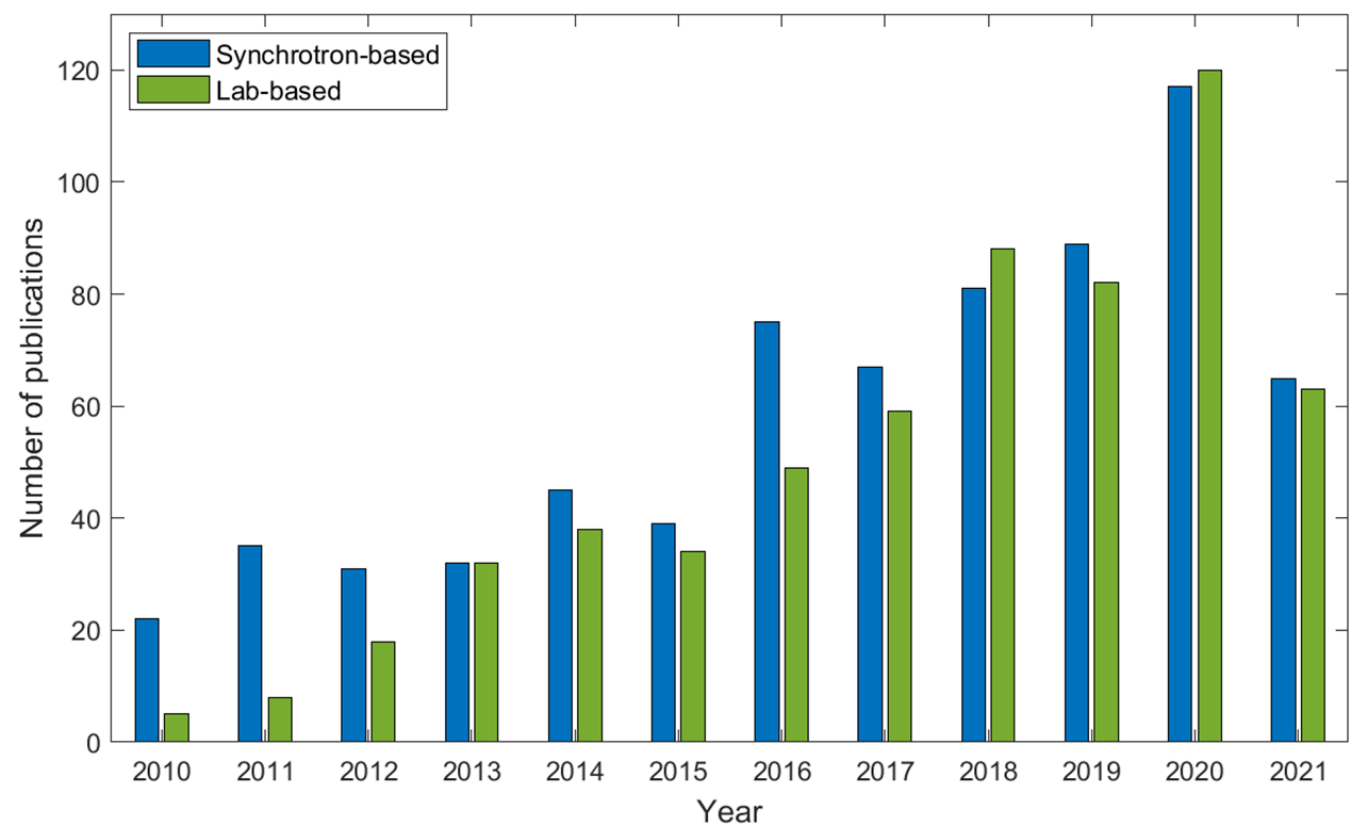

Figure 1. Publications on high speed and in-situ scanning over the last years separated for synchrotron and lab-based scanning obtained through querying Web of Science as detailed in the supplementary information (available online at stacks.iop.org/MST/33/012003/mmedia). 2021 data is incomplete, but includes publications up to July.

\subsection{High-throughput $C T$}

Before considering the reduction of a single object's acquisition time for high-throughput scanning, the challenges faced when exchanging samples in the field of view must be addressed. Bauza et al [45] showed that this can be very convenient in an industrial environment as stacking can, for example, be automated with a multipart fixture. However the size, shape and material of the object can impact the viability of this method, e.g. stacking is limited by the machine size and required voxel size, or could damage samples. The stacked objects do not need to be exactly identical since the settings can be selected on a per-scan basis. For example Hipsley et al [46] packaged a large number of small fossils in such manner that $3 \mathrm{D}$ volumes were obtained for every fossil in just three stacked scans. This decreased the total scan time significantly but this method is only useful for a limited number of academic users, thus automatic sample changes are desired. Some manufacturers have systems specific solutions such as ZEISS' autoloader module that allows loading up to 14 samples [47]. Eberhorn et al [48] propose another automated handling concept for inline XCT, redesigning the lab-XCT setup by adding an industrial robot that picks up a sample and then holds it in scan position, thus combining the loading and measurement procedures and has been employed by numerous manufacturers.

Some authors have identified the system geometry as the constraining factor to achieve high sample throughput and have demonstrated some novel approaches that utilise conveyor belts as shown in figure 2. De Schryver et al [49] rotate the object at the same time it travels past a static source and detector, scanning and performing an exact mathematical reconstruction. This geometry allows for a higher throughput but at the cost of detectable feature size (about $0.7 \mathrm{~mm}$ ) caused by increased blurring due to movement; a higher throughput, and thus faster scanning, means a lower resolution. Moving away from a static source and detector as in conventional labbased micro-CT an alternative gantry-style option is inspired by medical CT machines, as in figure 2(b). Here, the conveyor belt has a source and detector moving around it at highspeed in a helical trajectory [50]. Again, the detectable feature size is much larger than in typical micro-CT machines due to the source-object and source-detector distance being fixed to accommodate a multitude of samples. Warnett et al [51] explore the use of a real time tomography system similar to an airport baggage scanner for metrology applications in AM as in figure 2(c). This geometry consists of a conveyor belt but with a stationary ring of $900 \mathrm{x}$-ray sources that are individually switched and stationary rings of detectors. They show this geometry has potential, but the application are limited once again due to the resolution.

Another important aspect of high-throughput focuses on real-time reconstruction where data is reconstructed as received, and is seen as essential for automation in inline quality control. Buurlage et al [52] developed a method to reconstruct only critical slices, which is faster than reconstructing the whole $3 \mathrm{D}$ volume as is conventional. This certainly allows for real-time inspection, but is only applicable in certain circumstances such as when known features are expected in a known area of interest. Janssens et al [53] propose a neural-network Hilbert transform to be able to perform fast inline X-ray inspection where limited-angle projection data is directly transformed into a 3D volume. They show their algorithm is faster than the conventional filtered back projection (FBP) reconstruction by scanning walnuts in a conveyor belt geometry and reconstructing good quality images extremely fast-within $200 \mathrm{~ms}$. However, the training time 

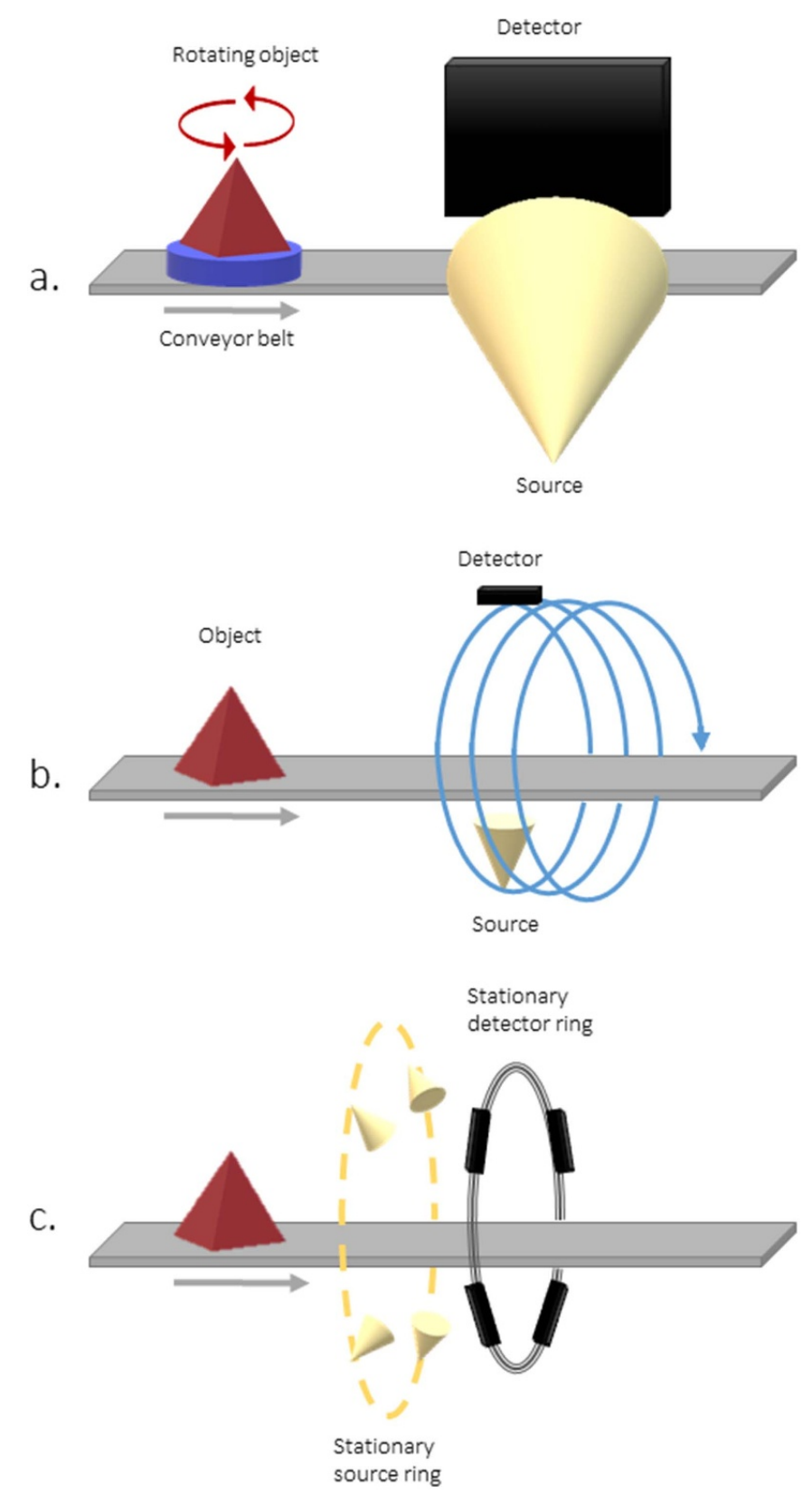

Figure 2. Different geometries for high-throughput CT with figures adapted from (a) De Schryver et al [49], (b) Brunke et al [50] and (c) Warnett et al [51].

needed for the algorithm means this method is most suitable for high-throughput testing of similar objects. Körner et al [54] reduced the number of projections by interpolating the missing information from the sinograms and looked at the effect on topography measurements. They showed this method has potential to provide the needed information when reducing the required number of projections by up to $60 \%$. This method shows potential but their study is limited to a single material cube, with a more tangible understanding of resolution impacts still required. The drawback of all the examples mentioned above is that they can only be used in specialist circumstances or have a low resolution.

\subsection{In-situ}

In-situ CT indicates the scanning of a process inside the CT machine, with the details of acquiring before and after or during loading discussed in detail in Gajjar et al [55]. Synonyms such as 4DCT (where the additional dimension is time), temporal, time-lapse and time resolved imaging are also used but all refer to a sequence of volumetric scans over a time period that can be of the order of seconds, minutes, hours of even days. The frequency and duration of each scan time depends on the process; some processes require the study of images every few seconds, while a scan per day is sufficient for other phenomena. Acquisition while the loading is occurring is relatively new for lab-CT [29], and continues to add to the confusing diction-referred to specifically as 'continuous' or 'dynamic' scanning. Figure 3 gives an overview of the different terms. As indicated, in-situ does not necessarily mean fast acquisition but there is a clearly a (large) subset of such experiments where speed is of the essence, for example to observe the changing morphology of an operating lithium battery [56] or pore-scale experiments [31,57]. Advancing our capability of high-speed scanning could allow for an even greater range of in-situ experiments and further detail on phenomena with additional discrete time points observed in the same period. Specifically lab-based developments are discussed here, with details on in-situ synchrotron scanning found in Beckmann et al [58].

Lab-based in-situ CT started in the early nineties with the first published examples in [59-61], with Breunig et al [59] proposing a 'simple load frame' to study fatigue cracks. This was complemented by Bay et al developing an analysis technique called digital volume correlation [62] that allows for 3D strain calculation and deformation measurement of image stacks achieved through in-situ imaging with numerous examples found in literature [63-68]. The range of applications in lab-based in-situ research is as broad as for regular CT, with figure 4 depicting examples with different time resolutions. In the life sciences it can be used to track growth which often takes days: Blunk et al [69] study the germination process of seeds, Lowe et al [70] the metamorphosis of an insect and Keyes et al [71] the deformation of soil caused by growing plant roots. Porosity (like) measurements are also performed in in-situ settings but in a range of different applications and time scales. For example, the slow processes of the failure mechanism of cementious synthetic foams [72] and the shrinkage of foam concrete due to drying [73]. In these cases, one or less scan a day is sufficient to processes whereas fast scanning was required for situations such as the baking of pastry and the deformation of beer foam [29]. In micromechanics labbased in-situ CT experiments have many applications: from experiments on the tension loading of glass-fibre reinforced composited [74] to corrosion damage [75], but currently many are still performed at synchrotron sources as the processes need shorter exposure times. Vavřík et al [76] showed that they can reach an exposure time of $3.4 \mathrm{~ms}$ with lab-equipment which already would allow for more dynamic processes to be imaged with 4DCT. Users are of course limited by the frame rate of their detector and require an entirely different 


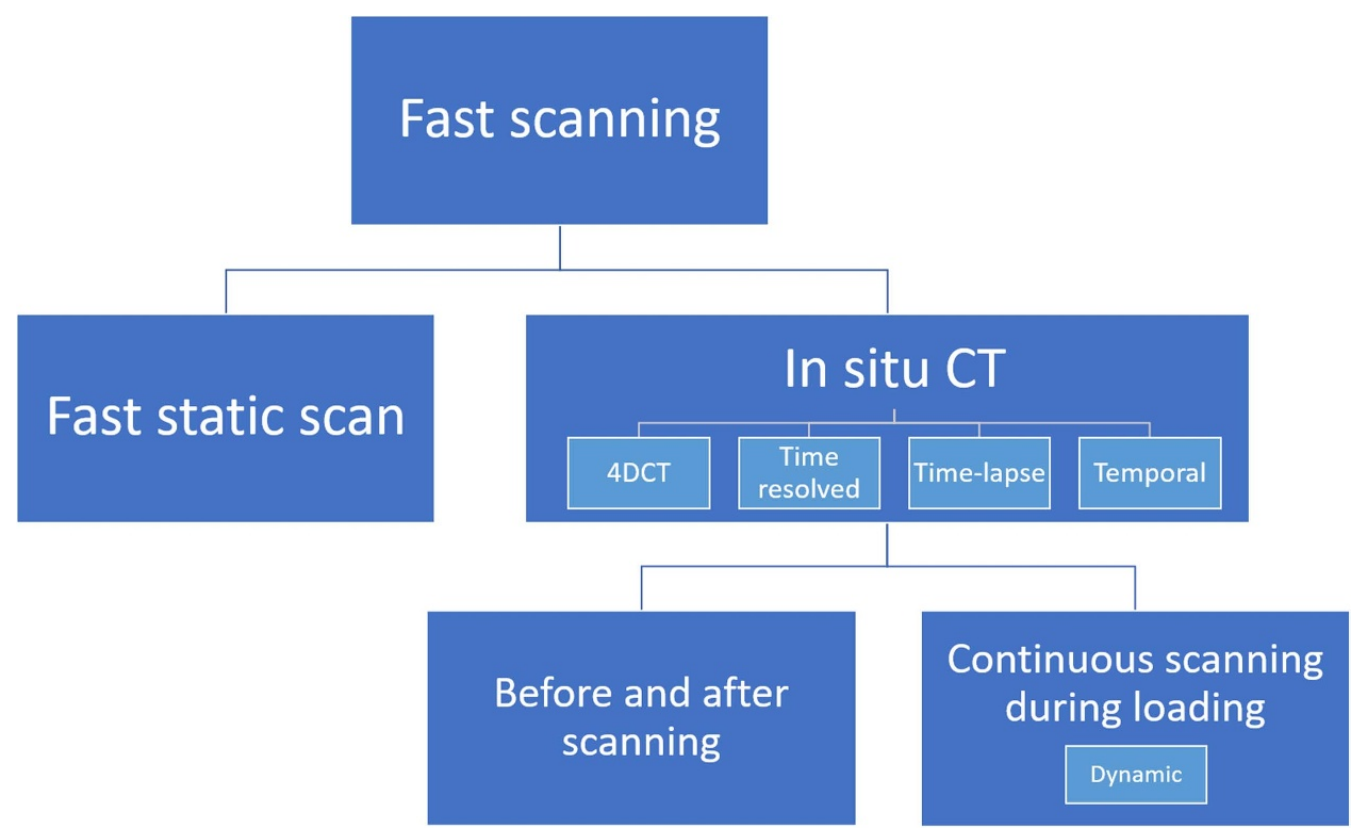

Figure 3. Diagram with different terms that can indicate fast scanning.

perspective to the setup than static objects where scan duration is less of a consideration. The next section will therefore focus on the parameters that (indirectly) affect the acquisition time.

\section{Acquisition parameters}

CT scanning consists of three stages: acquisition, reconstruction and analysis. It starts with the acquisition where the object is placed on a stage between an X-ray source and a detector. During a standard acquisition process the stage rotates while the detector captures projections at a number of angles. The parameters the user can set are: magnification, source power, voltage, exposure time, the addition of physical filters, binning, averaging, the number of projections and scan mode. In order to obtain a volumetric image for analysis the projections need to be mathematically reconstructed. This is often performed with software from the machine supplier that has an FBP/FDK algorithm implementation [79] although further relevant discussion can be found in the conclusions. Making an accurate estimation of the measurement error is important for dimensional measurements, which should include those arising in the scan process. These can be grouped in five main categories as seen in figure 5: CT system, data processing, the test object, environment and operator. Their influence has been studied by many authors, for example the effect of geometrical misalignments [80-83] and the surface of the test object $[84,85]$. Hiller et al [86] investigated the physical characterization and performance of the $\mathrm{x}$-ray tube and detector. Villarraga-Gómez et al [87] considered the significance of temperature and the bias determination. Lifton et al [88] studied the influence of beam hardening and scatter for dimensional measurement. And Rodríguez-Sánchez et al [43] address the understanding of the contribution noise has on the measurement uncertainty. Also the effects of processing should be taken in to account: Bartscher et al [89] wrote about the effect of data filtering and Stolfi et al [90] quantified the contribution of post-processing.

In order to acquire a scan an operator has to choose a set of acquisition parameters that ultimately influences the acquisition time and image quality. There is no analytical method to determine the optimal scanning parameters [92], but there have been attempts at making the process more automatic [93]. The BSI BS EN ISO 15708 [32] and VDI/VDE 2630 [33] standards do not provide a protocol for parameter selection, leading to differences in acquisition parameters between operators for the same object [40]. Experienced users will be aware of the different acquisition parameters but not all will be aware of the impact they have on their images. This section will explain the different parameters and their effects on the reconstructed image, leading to a discussion on how people are (should) choose their parameters to decrease the acquisition time in the subsequent section. To provide context, the workflow in figure 6 has been provided by experienced users of CIMAT, University of Warwick, describing the different steps of setting the parameters to obtain an optimal image while minimizing the acquisition time.

\subsection{Magnification}

The first step is to align the object within the field-of-view and set the magnification, and thus voxel size. The voxel size is often confused with spatial resolution; a voxel is the size of the smallest element in a volume (image stack) with an associated grey value, whereas the spatial resolution is a measure of the size of the smallest observable object within a scan which will be made up of a number of voxels [94]. Spatial resolution is defined as the smallest separation at which two features can 


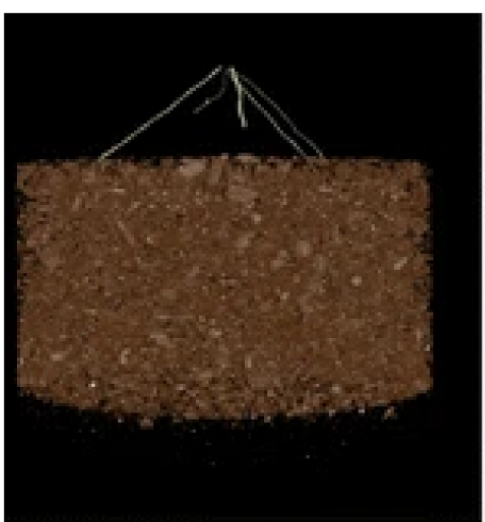

Week-4

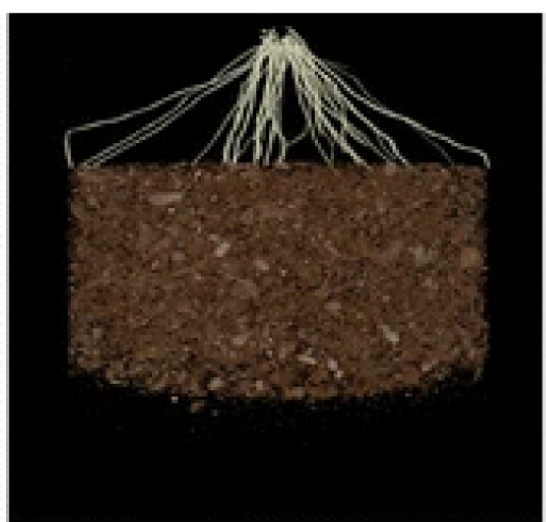

Week-8

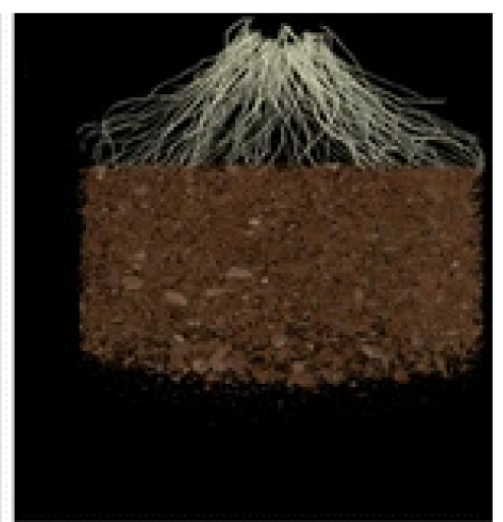

Week-12

(a)
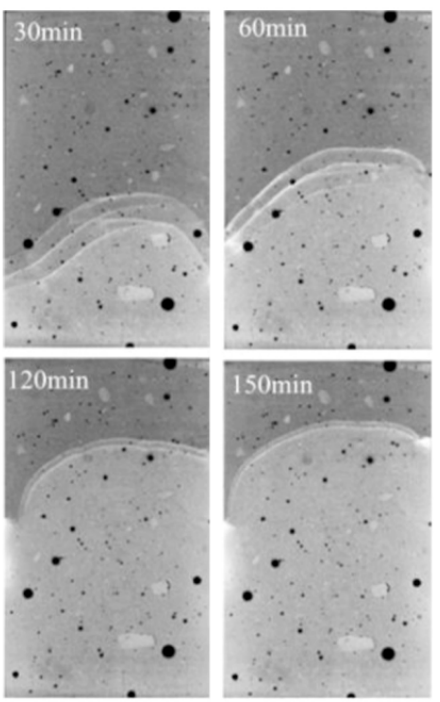

(b)

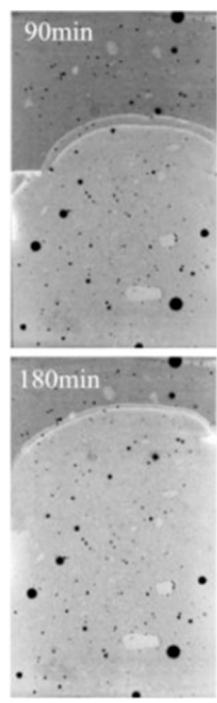

(b)

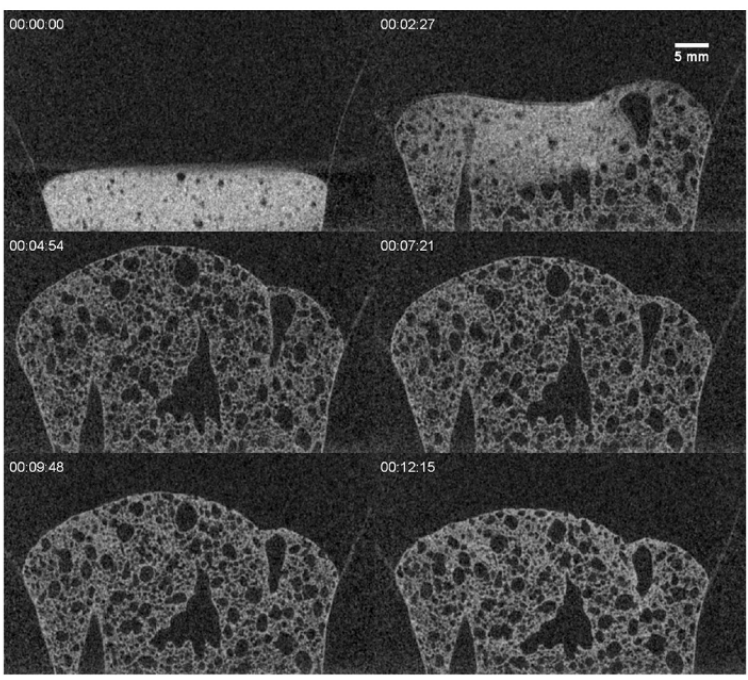

(c)

Figure 4. Examples of in-situ CT imaging: (a) changes over time of a root system, a time scale of weeks. Reprinted from [77], Copyright (2013), with permission from Elsevier. (b) Water transport in cement paste, half an hour between images. Reprinted from [78], Copyright (2015), with permission from Elsevier and (c) baking process of a muffin with a time resolution of 11.3 s. [29] John Wiley \& Sons. (C) 2020 The Authors Journal of Microscopy (C) 2020 Royal Microscopical Society.

be distinguished as separate entities [32]. The magnification is given by:

$$
M=\mathrm{SDD} / \mathrm{SOD} .
$$

This formula shows that the same magnification can be achieved using different source-to-detector distances (SDDs) as long as the source-to-object distance is changed accordingly. The physical dimensions of the machine restrict the minimum and maximum values for these distances; there is a limit to how close the object can be to the source while it can still turn without collision and, if the detector can be moved, it can only be moved as far as the end of the measurement chamber of the machine. In the case that both the detector and object can be moved relative to the source, an operator should pay attention to the chosen distances as they have influence on the noise in the image. A smaller SDD will give a brighter projection image as it follows the inverse square law for intensity. This means if the distance halves the brightness will be four times greater and therefore a much faster acquisition time, although the effect of cone beam artefacts could increase [95].

\subsection{Voltage and power}

After aligning the object an initial voltage of the $\mathrm{x}$-ray source has to be set; a guideline might be $80 \mathrm{kV}$ for light materials such as polymers and $160 \mathrm{kV}$ for heavy materials or larger objects [96]. The voltage gives the maximum energy of the polychromatic spectrum whereas the mean energy produced will in fact be much lower, adjusted for in the next step. Then the operator should set the tube power in such way that the spot size is similar to the voxel size. The focal spot size is defined as the x-ray emitting area on the anode of the x-ray tube as seen 


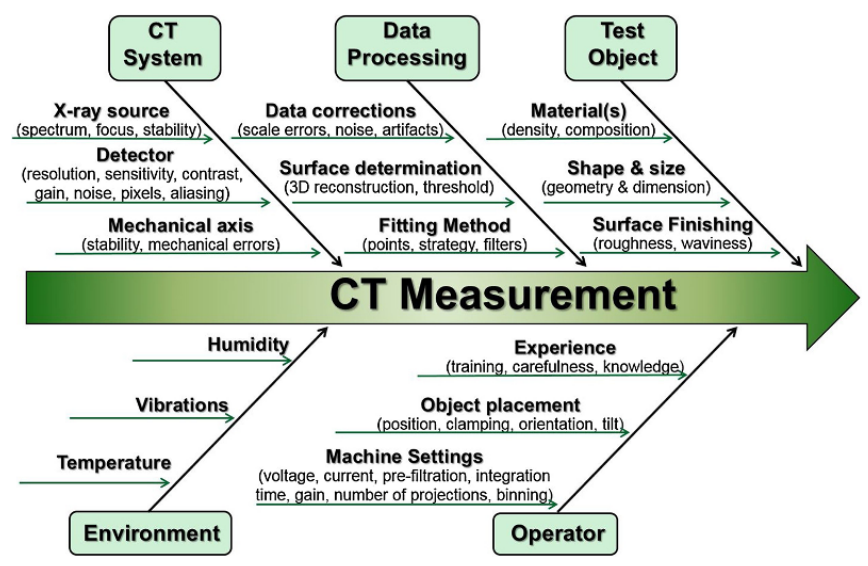

Figure 5. Ishikawa diagram depicting some of the main influencing factors for CT measurement. Reprinted from [91], Copyright (2018), with permission from Elsevier.

from the measuring device [97]. A higher power increases the intensity of the electron beam, increasing the heat in the focal point and thus increases the spot size. Power is therefore limiting to spatial resolution as a larger focal spot increases the Penumbra effect otherwise known as unsharpness. This effect causes blur at the sample edges in the image which becomes 'noticeable' when the spot size exceeds the voxel size $[98,99]$. It is important to maximise the power using this rule as a higher power increases the number of $\mathrm{x}$-ray photons per unit time and therefore decreases the acquisition time, but keeping it within the limit will minimise the Penumbra effect. For an overwhelming majority of $\mathrm{x}$-ray sources a rule of thumb is that the spot size increases by 1 micron for every Watt, but there are a few that do not; one should always obtain this information from their system manufacturer, or even better measure it themselves. One can measure the spot size of their particular setup by evaluating the line profile of a platinum wire or tungsten sphere as detailed in BS EN 12543 [97].

\subsection{Exposure time, voltage and filter}

The exposure time, voltage and filters are used to optimise the brightness and contrast of the image; maximising a broad histogram of grey values within the dynamic range of the detector and the safety limits of the system is preferred for optimal image quality. The exposure time is proportional to the number of photons detected per projection, with a longer exposure meaning a brighter projection image and lower noise at the cost of a longer acquisition time.

If the minimum grey value count is too high or too low, the voltage can be adjusted or the filter can be changed. It can be a difficult task to choose the optimal combination of voltage and filter for a specific measurement [101]. Figure 7 shows exemplar spectrums with different voltages and filters where the increase in mean energy and decrease in intensity can readily be seen to motivate the following discussion. The voltage sets the maximum energy of the spectrum where the mean energy is actually much lower. Filters increase the mean energy of the spectrum produced as they absorb lower energy
$\mathrm{X}$-rays, with the resulting spectrum having an improved penetration. This increased penetration (which maybe required) comes at the expense of a lower intensity of the spectrum due to this filter absorption. With the initial voltage selected without the use of a filter, there are two possibilities; the minimum grey value count is either too low or it is too high. If the minimum grey value count is too low, the voltage should be increased or a filter should be added/increased in thickness to allow greater penetration as per the examples in figure 7 . The converse of this is true when the minimum grey value count is too high. This balancing influences the mean energy of the spectrum, which is also largely responsible for the contrast; the lower the mean energy the better the contrast.

\subsection{Number of projections}

The last step is to set the number of projections, preferably according to the Nyquist sampling criterion found in signal processing [102]. It dictates that the minimum number of projections should be;

$$
\frac{\pi}{2} *<\text { window size }>
$$

where the window size is the width of the detector in pixels. This formula is based on the theoretical amount of information needed for a faithful reconstruction of the image. VillarragaGómez and Smith [103] examined the effect of under sampling on image quality and dimensional measurements; they concluded that while the image quality decreased the accuracy of the dimensional measurements did not decrease, although this was not the case for form measurements such as flatness and cylindricity.

\subsection{Other parameters}

There are some final considerations for more experienced operators. One setting is whether to have continuous acquisition or stepwise rotation acquisition. In other words acquisition with continuous rotation and taking projections or stopping rotation at equal intervals to acquire an projection. The latter option takes approximately 1.7 times longer, but arguably a reduced the presence of motion artefacts. Another option is to take multiple projections at every angle and average them into a single projection (hence called averaging), which notably reduces the impact of noise. A final common option is binning. In this process, a square of pixels, typically $2 \times 2$ or $3 \times 3$, are combined into one pixel resulting in a proportionally brighter image $(\times 4$ or $\times 9$ respectively) as the signal intensities are summed. It comes at the cost of spatial resolution as the effective pixel/voxel size is increased (doubled or tripled), but the increased brightness of the image allows for a proportionally lower exposure time leading to a faster acquisition. Often binning does allow for even shorter minimum exposures to be set within the system as it is frequently defined by data transfer speeds of the detector, which could be particularly attractive in the context of fast scanning. 


\section{Acquisition protocol}

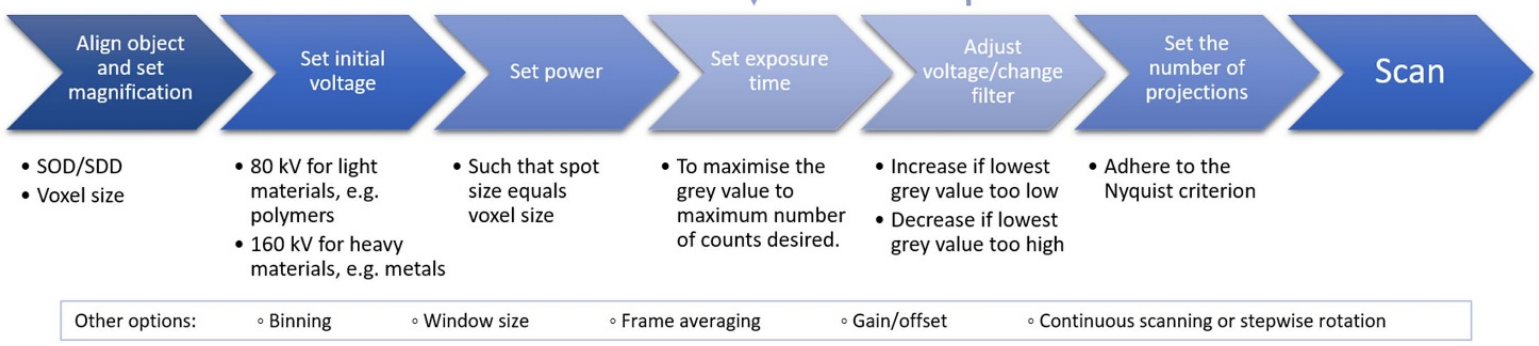

Figure 6. Acquisition protocol as provided by the experienced operators at CIMAT, WMG, University of Warwick.

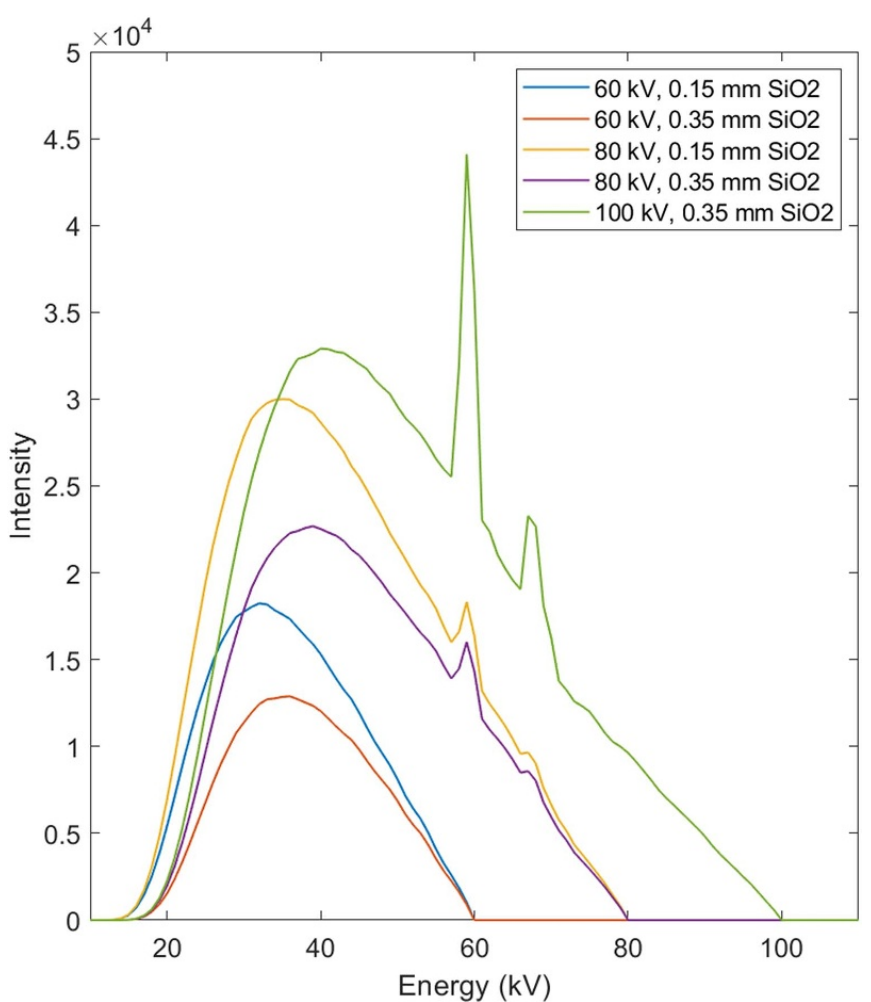

Figure 7. Examples of different spectra to demonstrate the impact of voltage and filter selection, generated with Spektr 3.0 toolkit [100].

\subsection{How to scan faster}

Theoretically, the acquisition duration is:

acquisition time $=$ exposure time $* \#$ of projections.

This assumes the object's rotation is continuous and no time is required for data storage. For example if the object stops turning for every projection the acquisition time should be approximately multiplied by 1.7 and could be included in the application, but is clearly impractical in the context of speed. There are therefore two options; lowering the exposure time or decreasing the number of projections.

First lowering the exposure time will decrease the number of photons per projection and thus lowering brightness.
If one is to maintain the dynamic range, this can be compensated by increasing the power since this increases the photon rate. As a higher power also gives a larger spot size this could decrease the sharpness of the image, which is shown in figure 8. Intentionally extreme cases are shown to observe the unsharpness and it is application dependent for what is suitable, but for qualitative purposes the potential acquisition speed gains can be significant. The second option is to use binning where, for example, four pixels are combined as one. The advantage of this is a four fold increase in brightness, at the cost of a lower resolution. The third option is to decrease the SDD as it increases the number of photons that reach the detector, but could be mechanically limited (impossible) by the machine. The fourth option is to set a higher voltage, the increase of brightness comes at a cost of a reduced contrast. Figure 9 shows a two-phase scanned object with increased voltage and different filter thicknesses to illustrate this, examples of the corresponding spectra can be found in figure 7 . One could also not compensate for the lower exposure time and accept a lower dynamic range, but this would lead to an image with lower contrast and more noise.

The number of projections that should be acquired is defined by the Nyquist criterion. In order to decrease the number of projections without violating the Nyquist criterion the window size could be decreased. This can be achieved by binning, this effectively halves the window size and in that way the required number of projections is halved, again, at the expense of a lower resolution. To be explicit, binning has two effects in terms of acquisition time, both on the exposure time and the number of projections, thus has the most significant influence across all considerations. A less impactful approach to reduce the effective window size would be to adjust the magnification allowing more gentle reductions rather than $50 \%$ smaller, but while you reduce the number of projections you do not achieve the brightness benefit of binning pixels. One could also not adhere to the Nyquist criterion and reduce the number of projections without changing the window size. Figure 10 shows scans of the same object with different numbers of projections. It can be observed that a lower number of projections results in more noise and artefacts, but again modest reductions could be sufficient without decreasing measurement accuracy. 


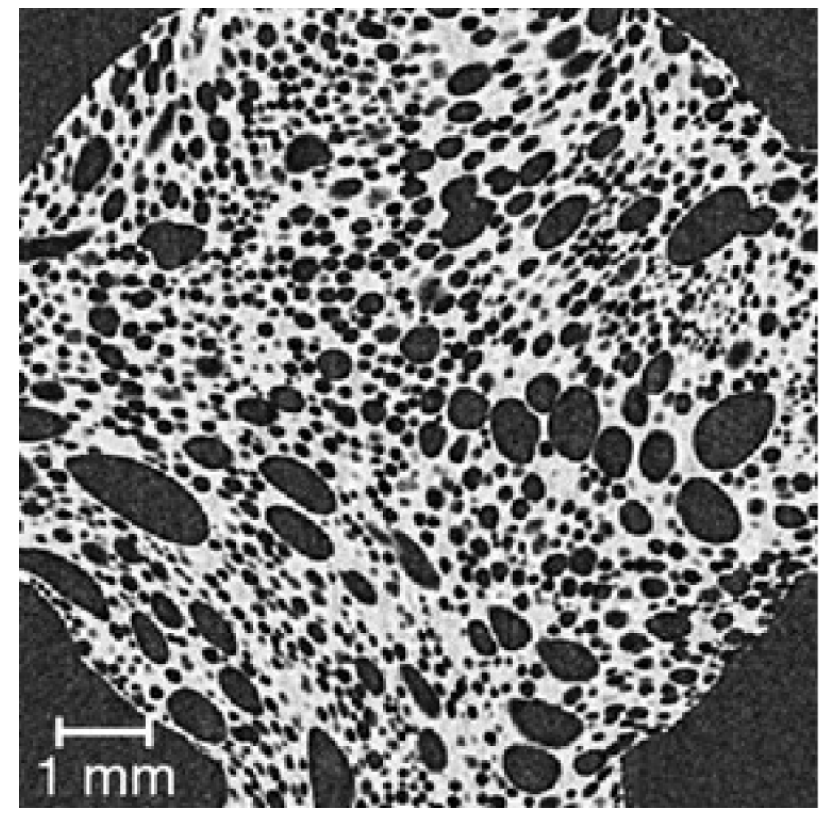

(a) $25 \mu \mathrm{m}$

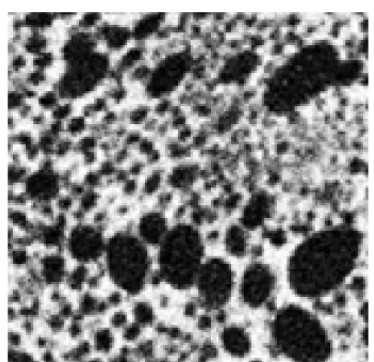

(b) $50 \mu \mathrm{m}$

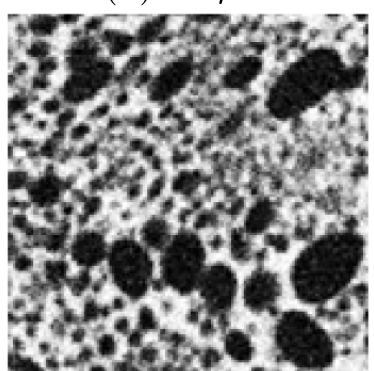

(d) $100 \mu \mathrm{m}$

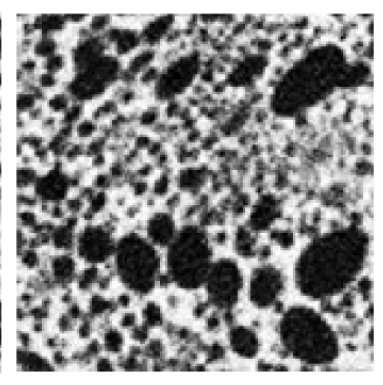

(c) $75 \mu \mathrm{m}$

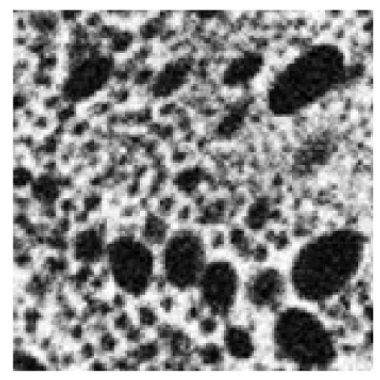

(e) $125 \mu \mathrm{m}$

Figure 8. Reconstructed images of a foam acquired using the TESCAN UniTom XL with a voxel size of $25 \mu \mathrm{m}$, fixed $\mathrm{kV}$ and projection number, but increasing power and therefore spot size leading to reduced acquisition times. (a) $25 \mathrm{~W}$. Standard spot size of $25 \mu \mathrm{m}$, duration 3016 s. (b) 50 W. $50 \mu \mathrm{m}$ spot, duration 1598 s. (c) 75 W. $75 \mu \mathrm{m}$ spot, duration 1101 s. (d) 100 W. $100 \mu \mathrm{m}$ spot, duration 845 s. (e) 125 W. $125 \mu \mathrm{m}$ spot, duration $694 \mathrm{~s}$.

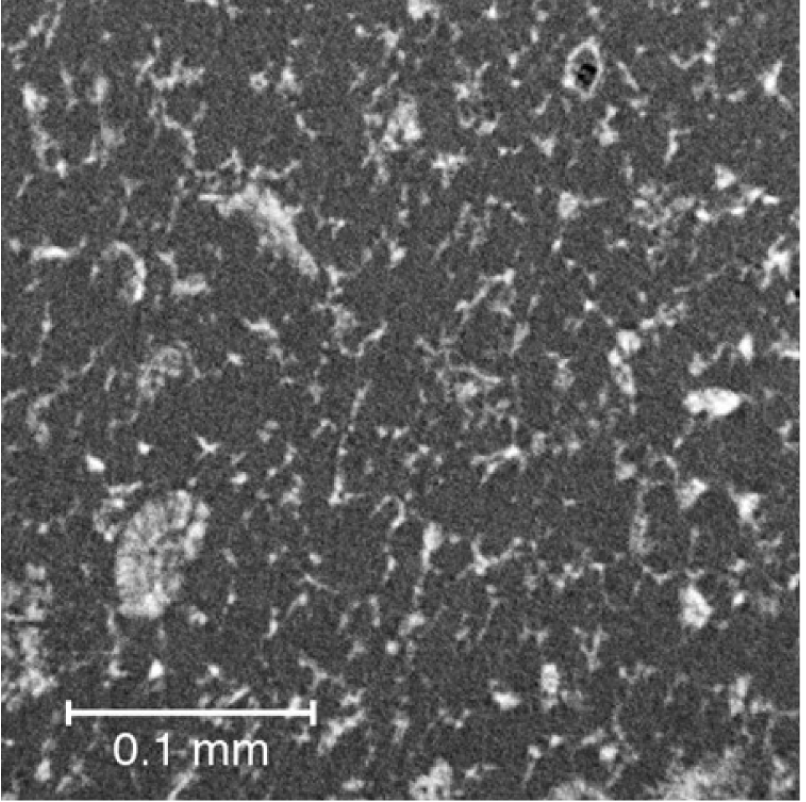

(a) $60 \mathrm{kV}, 0.35 \mathrm{~mm}$

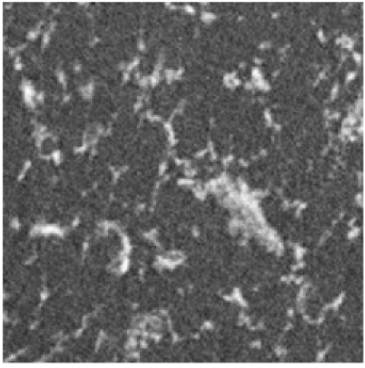

(b) $60 \mathrm{kV}, 0.15 \mathrm{~mm}$

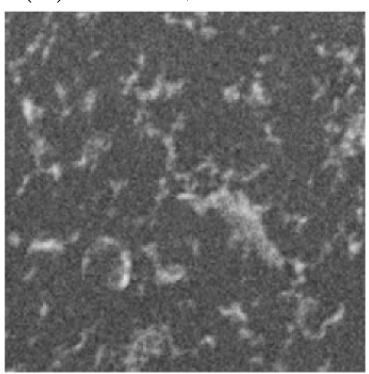

(d) $80 \mathrm{kV}, 0.35 \mathrm{~mm}$

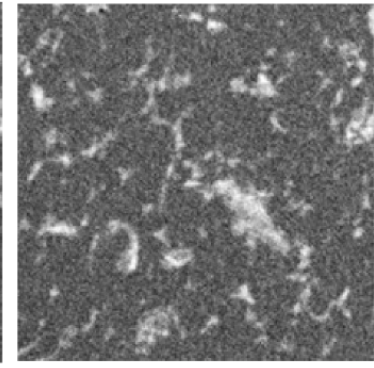

(c) $80 \mathrm{kV}, 0.15 \mathrm{~mm}$

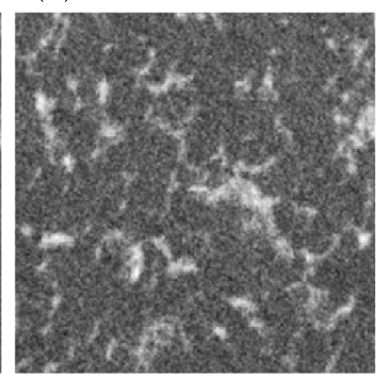

(e) $100 \mathrm{kV}, 0.35 \mathrm{~mm}$

Figure 9. Reconstructed images of an AlSi matrix with TiB2 particles acquired using the ZEISS Xradia Versa 620 with a voxel size of $0.4 \mu \mathrm{m}$, fixed power and projection number, but increasing voltage and different thickness glass $\left(\mathrm{SiO}_{2}\right)$ filters. (a) $60 \mathrm{kV}$ and $0.35 \mathrm{~mm}$ filter (standard), duration $12 \mathrm{~h} 25 \mathrm{~min}$. (b) $60 \mathrm{kV}$ and $0.15 \mathrm{~mm}$ filter, duration $11 \mathrm{~h} 34 \mathrm{~min}$. (c) $80 \mathrm{kV}$ and $0.15 \mathrm{~mm}$ filter, duration $9 \mathrm{~h} 51 \mathrm{~min}$. (d) $80 \mathrm{kV}$ and $0.35 \mathrm{~mm}$ filter, duration $10 \mathrm{~h} 43 \mathrm{~min}$. (e) $100 \mathrm{kV}$ and $0.35 \mathrm{~mm}$ filter, duration $9 \mathrm{~h} 0 \mathrm{~min}$. 


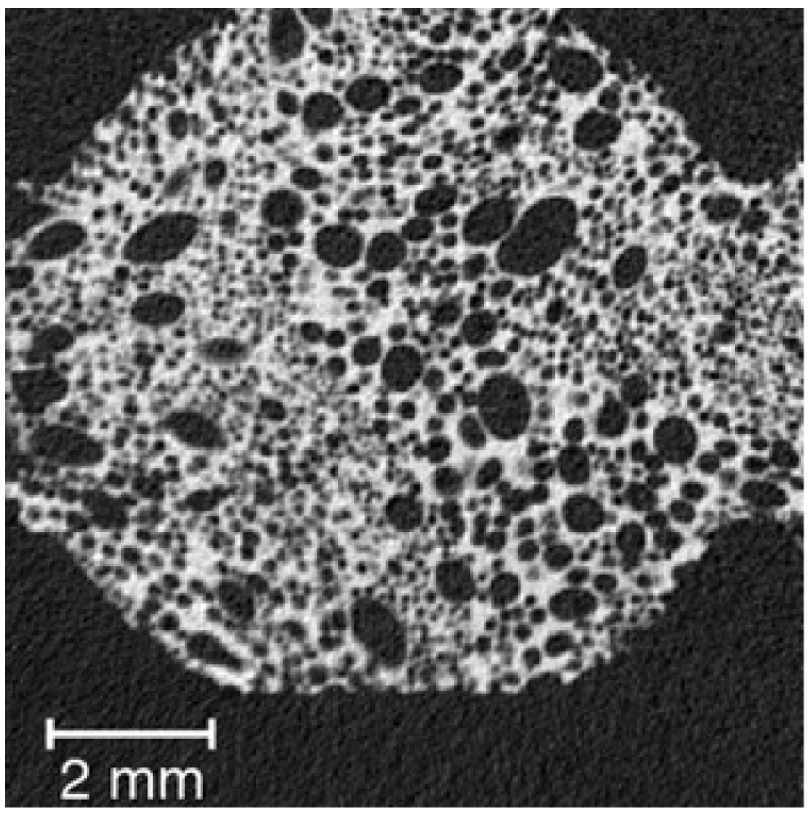

(a) $100 \%$

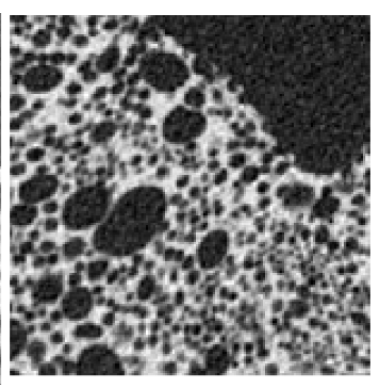

(b) $80 \%$

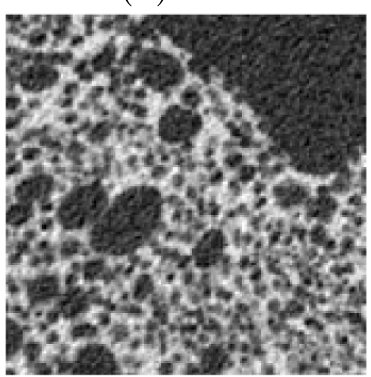

(d) $40 \%$

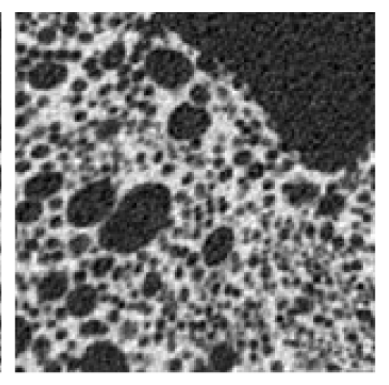

(c) $60 \%$

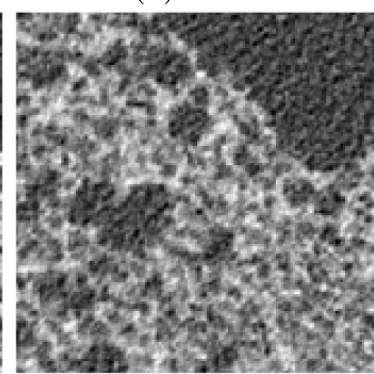

(e) $20 \%$

Figure 10. Reconstructed images of a foam acquired using TESCAN UniTom XL with a voxel size of $25 \mu \mathrm{m}$, fixed $\mathrm{kV}$ and power, but increasingly reduced projection numbers as a percentage of the Nyquist sampling rule. (a) $100 \%$ (3016 projections), duration $346 \mathrm{~s}$. (b) $80 \%$ (2412 projections), duration 277 s. (c) $60 \%$ (1810 projections), duration 208 s. (d) $40 \%$ (1206 projections), duration 138 s. (e) $20 \%$ (603 projections), duration $69 \mathrm{~s}$.

\section{Evaluation of current parameter use}

This section will study what acquisition settings are currently being used for higher-speed acquisition and evaluate how they might be improved. Presented is a critical review of publications that utilised high-speed lab-based XCT across diverse fields from 2010 to 2021, with the exact search and resulting articles presented in the supplementary information. Most studies on faster scanning focus on the applications themselves and not how they approached parameter selection for the shortest acquisition time. This results in papers with limited information on their scanning parameters and lack reasoning (and sacrifices) behind selection. Another implication is that not all articles with fast scanning were found using the Web of Knowledge search but a balance was found between broad search terms and relevant results. The resulting papers were checked against our definition of fast and in-situ scanning discussed in the introduction, with the additional requirements being the use of a non-medical CT system set up and experimental results included in the paper. Following these restrictions a total of 596 journal articles were mined for scan parameters, after which a time limit of $1 \mathrm{~h}$ per scan was set for the parameter comparison.

The focus is on commercial machines as these are (more) accessible to non-expert users and easier to compare with publicly available information on the detector and source performance. A few publications that use in-house built machines with very fast acquisition times will be mentioned as those give an impression of the potential for dedicated high-speed systems. Figure 11 distinguishes the different manufacturers and acquisition times across the publications. ZEISS systems have been the most popular for high-speed and in-situ scanning with $50 \%$ more publications than any other machine, but clearly this largely comes from acquisitions greater than an hour. The authors propose two potential reasons; one might be the lower concern for scan speed and more the application for which these were sufficient, or alternatively it might be because they were an early adopter of integrating commercially available in-situ rigs (e.g. Deben) and making it easier for users to run these types of experiments. Another notable observation is that TESCAN has the highest proportion of sub 1 min acquisitions and overall their distribution is more weighted to the highest speed experiments. They have a much lower number of publications however which likely stems from their relatively later entry to market, with the earliest publication in 2016.

\subsection{Exposure time}

Table 1 gives an overview of the fastest acquisitions times performed on lab-based machines. Dewanckele et al [29] are clearly the fastest of the commercially available machines; their acquisition time is $9.4 \mathrm{~s}$. The only systems currently available on the market that can seemingly achieve these sorts of speeds are all made by TESCAN that has 4D scanning as their marketed unique selling point. Of the other system manufacturers, Nikon having been fast enough to achieve 1 min scans $[104,105]$, are more than twice as slow when purely considering exposure time. There are no ZEISS' scans in the table;4D scans have been performed with their machines, but predominantly on long processes with scans taking more than $1 \mathrm{~h}$ as already highlighted. For comparison, the typical minimum exposure times under $2000 \times 2000$ detector modes without 


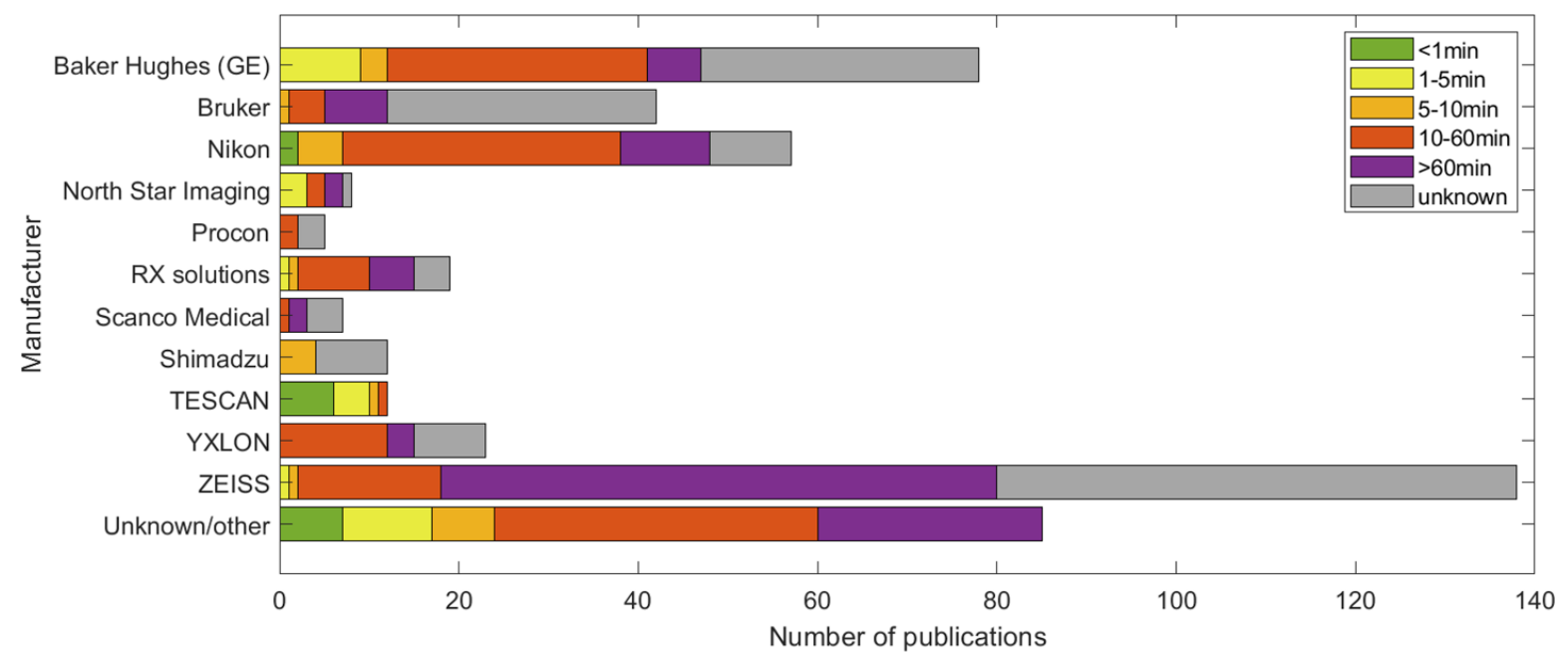

Figure 11. Overview of number of publication of the different commercially available XCT system manufacturers subdivided for acquisition time.

Table 1. Overview of the fastest scans performed on lab based systems as identified in literature search, sorted by acquisition time, similar publications with $12 \mathrm{~s}$ acquisition times can be found in [57, 106, 107].

\begin{tabular}{|c|c|c|c|c|c|c|c|c|c|}
\hline \multirow[b]{2}{*}{ Year } & \multirow[b]{2}{*}{ Author } & \multirow[b]{2}{*}{$\begin{array}{l}\text { Acquisition } \\
\text { time (s) }\end{array}$} & \multirow[b]{2}{*}{ Brand } & \multicolumn{6}{|c|}{ Parameters } \\
\hline & & & & $\begin{array}{l}\text { Exposure } \\
\text { time (s) }\end{array}$ & Projections & $\begin{array}{l}\text { Voltage } \\
(\mathrm{kV})\end{array}$ & Power (W) & $\begin{array}{l}\text { Detector } \\
\text { size }\end{array}$ & $\begin{array}{c}\text { Voxel } \\
\text { size }(\mu \mathrm{m})\end{array}$ \\
\hline 2017 & Vavř́k et al [76] & 1 & Custom system & 0.0034 & 252 & 60 & 50 & $256 \times 256$ & 40 \\
\hline 2014 & Eggert et al [108] & 8 & Custom system & 0.034 & 236 & 60 & 14.4 & $512 \times 512$ & 17 \\
\hline 2020 & $\begin{array}{l}\text { Dewanckele } \text { et al } \\
\text { [29] }\end{array}$ & 9.4 & TESCAN & 0.017 & 550 & 130 & 35 & $500 \times 400$ & 150 \\
\hline 2016 & Bultreys et al [31] & 12 & TESCAN & 0.02 & 600 & 130 & 16 & $657 \times 657$ & 14.8 \\
\hline
\end{tabular}

binning for a ZEISS Xradia machine is $1.0 \mathrm{~s}$, while Nikon can achieve $0.133 \mathrm{~s}$ and TESCAN $0.05 \mathrm{~s}$ (faster speeds can be achieved by binning as can be seen in table). These limitations on exposure time do not only depend on specifications of the system's detector and if useable under the setup, but they may also be further restricted by software to ensure quality scans as is the case for the ZEISS Versa. The detector can even vary between installations and is why further comment is not given whether a faster exposure could have been used, but current common flat panel installations are Varian/Perkin Elmer 1621 or 4343 (or variations thereof) if the reader wishes to find out more.

The exposure time influences the intensity received at the detector which is what one would ideally maximise for optimal image quality. Some systems allow shortening the SDD which enables users to reduce the exposure time while keeping the same photon intensity as described earlier. Another way to decrease the exposure time in all systems is by increasing the source power. This will increase the number of photons per unit time and thus a lower exposure time can be used. However this can cause increases in the spot size as discussed, eventually leading to a notable unsharpness.

Figure 12 shows the spot size $(\mu \mathrm{m})$ against voxel size $(\mu \mathrm{m})$ across different publications with acquisition times below $1 \mathrm{~h}$. The spot size within a specific study has been determined by relating the power stated to the spot size value provided for that source by the manufacturers. The dotted line indicates the most optimal situation with minimised unsharpness; the voxel size equal to the spot size. The expectation would be that the majority of the data points lie roughly along this line with maybe a few above where they have sacrificed quality for speed, but there are a notable number below the threshold. For those where the spot size is larger than voxel size, the authors have likely/hopefully identified that their image quality does not measurably suffer for their intended purpose. Note that ZEISS' Xradia operators will never violate the spot size requirement due to the software that maintains the spot size at 3 or 4 microns up to $10 \mathrm{~W}$; these studies account for a high proportion below the line. Experiments utilising Baker Hughes systems are almost all exceeding this limit, as are the few from North Star Imaging. Nikon based inspections are much more equally distributed either side of the line indicating than many here could improve their acquisition times, if it were beneficial, by increasing their power and thus reducing exposure and acquisition times. For the TESCAN based publications in table 1 and figure 12 it is interesting that the spot size could theoretically be increased as the voxel size of the scans is relatively large. However, in this system the source-detector geometry can be particularly compact so it is more likely that the maximum grey value count without saturating the detector was reached at the chosen exposure and binning.

Adjusting the power is not the only option when aiming to decrease the exposure time, but it is the option one can interrogate directly from information in the publications. 


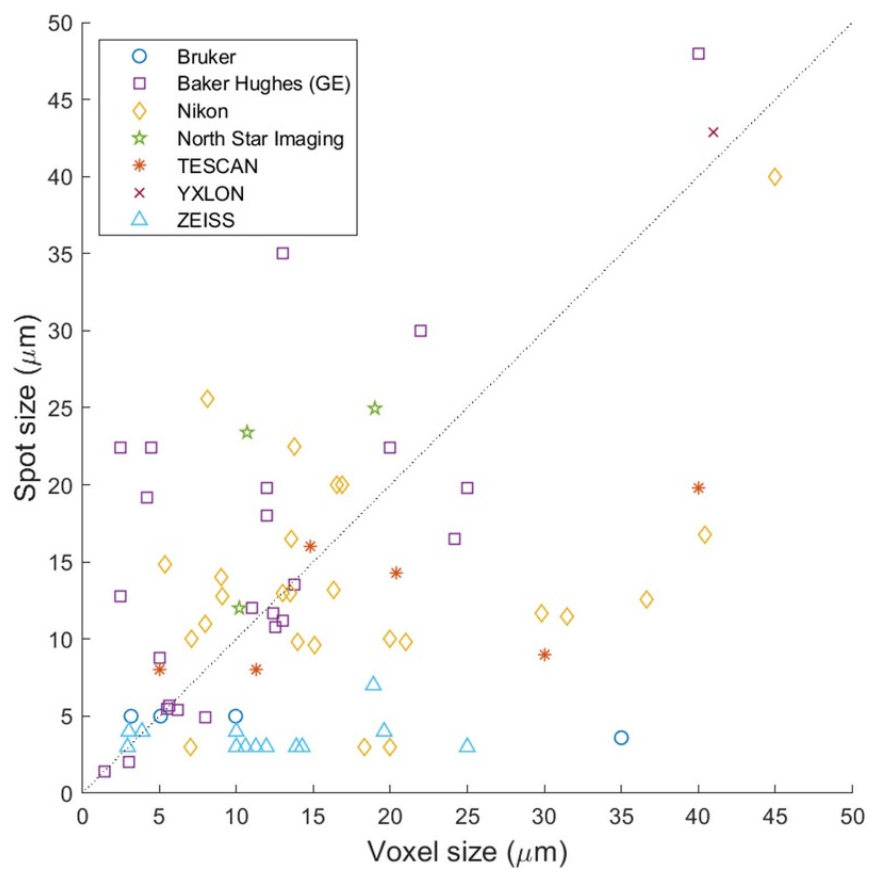

Figure 12. Spot size vs. voxel size across the identified publications. Spot size has been determined from manufacturer system materials and pers. comms. but may not reflect the calibration of the machine at the time.

Brightness can also be increased by setting a higher voltage as demonstrated earlier in figure 9 but this can reduce the contrast. Depending on the application this may not be critical and can therefore benefit a faster acquisition time. Additionally it is assumed studies used the whole dynamic range of the detector but this was not stated in any publication. Not using the full dynamic range results in darker projection images which will likely increase noise. Likewise the detector gain, an amplification of the signal received by the detector at a cost of increasing the noise, was largely not given by authors, but it is assumed the gain and therefore amplification was the maximum so the smallest exposure times were possible. As all the options to lower exposure time reduce image quality users need to assess which aspects they are willing to comprise for their application to minimise impact.

\subsection{Number of projections}

Instead of decreasing the exposure time for faster scans the alternative is to reduce the number of projections. For optimal image quality the number of projections should obey the Nyquist sampling rule when using FBP reconstruction methods. This is the reason why detector binning not only increases the brightness but has the added benefit of proportionally reducing the minimum number of projections.

Figure 13 shows the distribution of the percentages of Nyquist imposed projections across publications, once again sub-divided by acquisition time. Clearly most operators do not obey the Nyquist sampling rule when performing in-situ and high-speed scanning in the lab, favoured as the quickest and most impactful way of reducing acquisition times. In fact many do not have half of the recommended number of

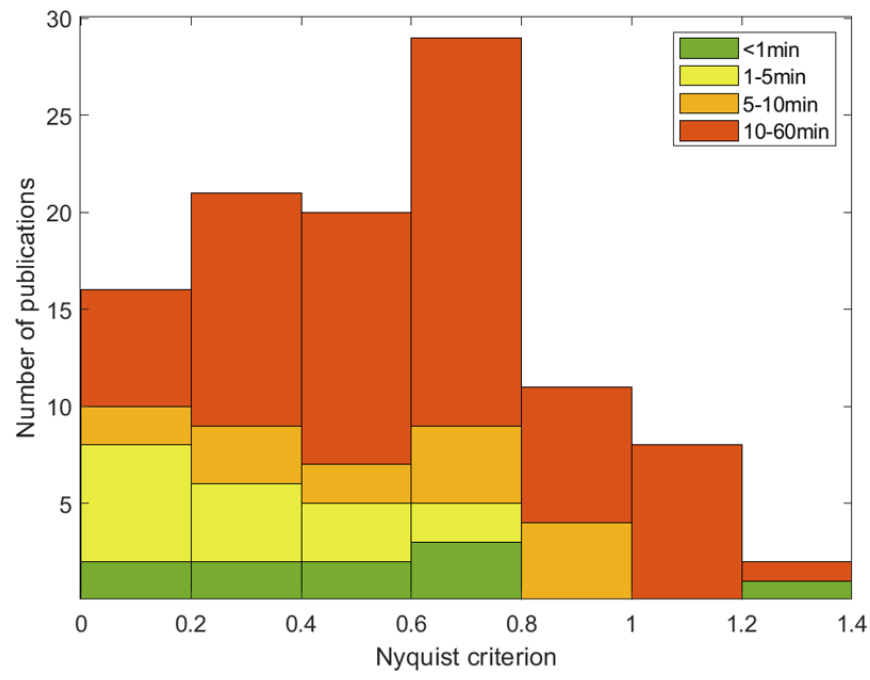

Figure 13. Histogram of the \# of projections/(window size $\times \frac{\pi}{2}$ ) for a single scan in the fast scans surveyed in the literature. When the number is 1 or higher the Nyquist sample rule is obeyed.

projections. The average is $60 \%$ and from the timing divisions, seemingly acquisitions less than $5 \mathrm{~min}$ are only viable then. One exception is a sub-minute (45 s) scan that actually over-sampled at $127 \%$ of Nyquist [109]. In this experiment they used a home-built micro-CT system to scan mice using an exposure time of $40 \mathrm{~ms}, 400$ projections and a resulting window size of 200 pixels after a binning factor of 4 . The small window size and fast detector rate allow for a sub-minute scan while still over-sampling with regards to the Nyquist criterion.

There are some remarkable cases of around 10\% such as Luksic et al [104] in their experiments characterising the foaming process during glass melting. While their parameter selection is not discussed it is clear the $58 \mathrm{~s}$ scans are out of necessity rather than choice since the whole experiment is 8 min long. While this results in significant image processing challenges as can be seen in figure 14 and the smaller features are questionably measured, the macro pores are clear and sufficient to gain insight into the process their task of understanding porosity changes with time.

All high-speed/in-situ publications included in figure 13 use FBP for fair comparative purposes, but there were 9 out of the 596 studies from the literature search that used iterative reconstruction methods that typically require a lower numbers of projections [55, 110-117]. For example Myers et al [113] experiments with two-phase fluid flow, used an iterative algorithm that exploits the a priori knowledge of the sample. This method reduced the number of projections from 720 in case of FBP to just 72 projections (window size $512 \times 512$ ) improving their time resolution by a factor of ten. Utilising novel reconstruction is more common place at the synchrotron but in lab-based high-speed/in-situ experiments it is currently a rarity.

\subsection{Summary}

High-speed and in-situ experiments in the lab have been performed in a variety of manufacturers systems. ZEISS has been 


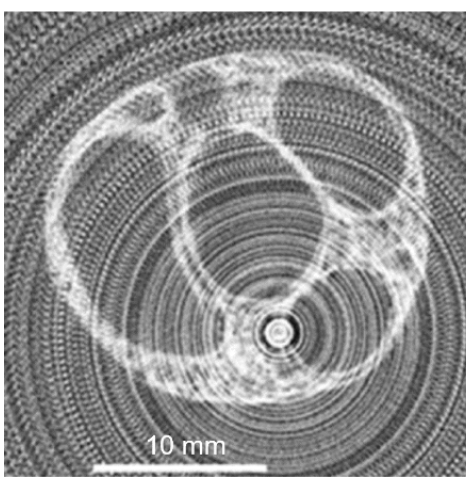

(a)

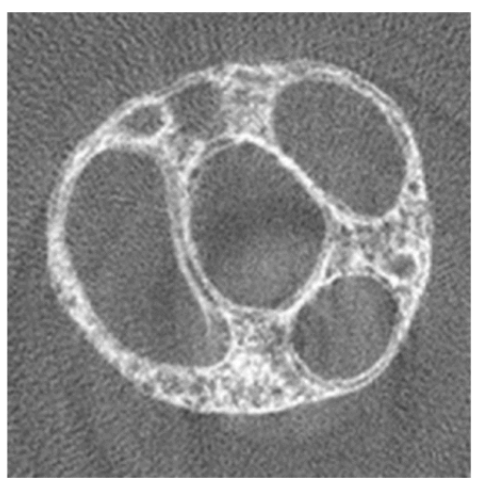

(b)

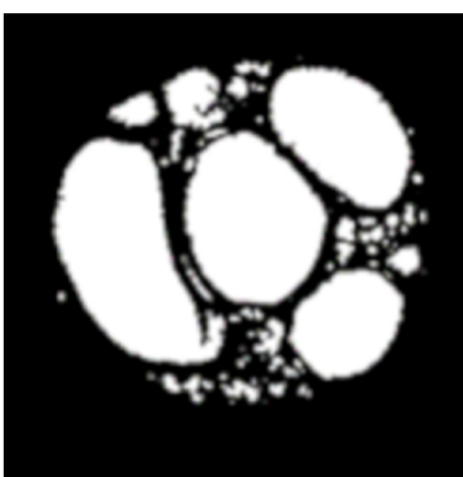

(c)

Figure 14. Experiment of foaming during glass melting [104] where around $10 \%$ of the required projections were used. (a) Initial raw reconstruction with image artefacts. (b) Repaired reconstruction. (c) Segmented bubbles within foam. Reprinted from [104], Copyright (2020), with permission from Elsevier.

the most popular system in this area over the period searched. The fastest acquisitions have been achieved on the TESCAN system, which the analysis indicates is due to the lower usable exposure times. Publications using this manufacturers systems do not comply with the Nyquist criterion, on average using $60 \%$ of the recommended projections, but this is comparable to others studies that utilised fast and in-situ scanning. It can be concluded that many operators can improve their scanning speed by increasing their spot size to match the voxel size therefore reducing exposure time without decreasing image quality, although this is not possible for every system due to both hardware and software. The Nyquist sample rule is not met by most operators, indicating that the recommended number of projections can be decreased while not impacting image quality for measurement and observation. These tweaks in acquisition protocol may still result in scan times that are too slow for the specific application which may have been the justification for even fewer projections and even greater spot sizes (increased power) identified, assuming the operator was familiar with the standardised parameter selection process. When users are making these decisions the final choices should always result in sufficient image quality for what they specifically want to observe and measure.

\section{Challenges and concluding remarks}

Driving the need for faster imaging with lab-based CT systems is a growing interest in in-situ experiments and a need for highthroughput resolutions that are fit for purpose. Currently most lab-based in-situ scanning is still relatively slow and the fastest acquisitions are limited to a few commercial machines and in-house built CT systems. In this review the parameters have been evaluated against a typical best practice protocol optimised for image quality and measurement accuracy within the shortest acquisition time.

Following this analysis it is evident that there is an inconsistent approach to deciding how to minimise the acquisition time of a scan for high-speed and in-situ scanning. From the comparisons and our own operation, it is recommended to:
- Maximise your spot size so it is similar to the voxel size. This will enable the fastest exposures while maintaining the optimal brightness and dynamic range.

- Binning. Most studies utilise this for high-speed since for $2 \times 2$ it results in an increase of brightness four times proportionally affecting the exposure, which often outweighs the impact of doubling the resolution. Further the number of projections can be halved leading to an eight times increase in scan time.

- Anecdotally, using about $60 \%$ of projection results in a workable image quality [103], but users should check this is suitable for their application.

These items have the greatest impact but there are a few more deviations from the standardised parameter selection to measurably reduce exposure. However, they have a detriment to measurement accuracy and image quality that currently is not quantifiable prior to acquisition.

- Higher energies $(\mathrm{kV})$ will slightly increase brightness allowing for a reduction in exposure time, but this can reduce the image contrast.

- Exceeding the power/spot size rule will proportionally increase brightness. This can increase unsharpness.

- Impose a lower than optimal exposure so the whole dynamic range is not used. This can increase noise and decrease contrast.

The impact on image quality and measurement is both sample and task specific, so to determine the optimal speed it is always advised to run a sensitivity study. The first steps on quantifying image quality have been taken by proposing metrics for CT [43, 44], but more research on image quality linked with fit for purpose is essential for creating fast scanning protocols for different applications.

Future hardware improvements could impact the acquisition speed without the need to compromise on image quality. Current developments of new sources will bring lab-based capabilities closer to synchrotron facilities. Gruse et al [118] 
demonstrated the application of laser driven sources that could be scaled down for industrial applications. These utilised 45 femtosecond pulses of $\mathrm{x}$-rays that could provide micron-scale resolution with fast scanning. Hornberger et al [119] presented the Compact Light Source that has a high average flux and brightness, but it is currently very expensive.

Minimising the amount of data to be acquired will have the greatest impact on acquisition speeds, as the advent of new hardware can be relatively slow but the algorithms to utilise less data are available now. There are a number of papers that show a fewer number of projections could be sufficient to obtain a suitable image quality and measurement accuracy by using a variety of iterative reconstruction methods [120-123]. However, most operators use FBP reconstruction methods because it is supplied by manufacturer. If operators want to use iterative reconstruction there is a level of coding they will have to implement through open-source software [124-127] making it appear as 'specialist' by typical operators and under-used, despite the advantages of faster acquisition times. Beyond iterative reconstruction machine learning methods have also shown promise in coping with less data [128], but again are currently 'expert/specialist' in their accessibility by the novice user. The current main disadvantage of iterative algorithms is that the required computational power is much larger and hence the time to reconstruct is much longer, although this time is reducing with the availability of ever more powerful GPUs. When considering a 'scan' as both the acquisition and reconstruction time the benefit gained of a faster acquisition with fewer projections might be negated by the duration of reconstruction; in the case of high-throughput this is the most impactful as immediacy of results is often required, but even in-situ experiments can suffer if one is unwilling to wait days/weeks/months for their data. This will likely change in the future as the availability of computational power is still rising fast. This shows both the interest in iterative reconstruction for in-situ measurements and the need for protocols based on the information required from a scan and again the great potential of iterative methods for reducing the number of projections.

\section{Data availability statement}

All data that support the findings of this study are included within the article (and any supplementary files).

\section{Acknowledgments}

The authors would like to acknowledge funding from EPSRC Strategic Equipment Award EP/S010076/1 and support from the National Research Facility in X-ray Computed Tomography Grant EP/T02593X/1. Further we would like to thank Samuel Harris for editorial support.

\section{ORCID iDs}

E A Zwanenburg (D) https://orcid.org/0000-0002-4776-5567
M A Williams (D) https://orcid.org/0000-0003-3802-7914

J M Warnett (D) https://orcid.org/0000-0003-2284-790X

\section{References}

[1] Alsop K, Baier W, Norman D, Burnett B and Williams M A 2021 Accurate prediction of saw blade thicknesses from false start measurements Forensic Sci. Int. 318110602

[2] Baiker-Sørensen M, Herlaar K, Keereweer I, Pauw-Vugts P and Visser R 2020 Interpol review of shoe and tool marks 2016-2019 Forensic Sci. Int.: Synergy 2 521-39

[3] Rutty G N, Brough A, Biggs M J P, Robinson C, Lawes S D A and Hainsworth S V 2013 The role of micro-computed tomography in forensic investigations Forensic Sci. Int. 225 60-66

[4] Baier W, Norman D G, Donnelly M J and Williams M A 2021 Forensic 3D printing from micro-CT for court useprocess validation Forensic Sci. Int. 318110560

[5] Cnudde V and Boone M N 2013 High-resolution x-ray computed tomography in geosciences: a review of the current technology and applications Earth-Sci. Rev. $1231-17$

[6] Kyle J R and Ketcham R A 2015 Application of high resolution $\mathrm{x}$-ray computed tomography to mineral deposit origin, evaluation, and processing Ore Geol. Rev. 65 821-39

[7] Lei L, Seol Y and Jarvis K 2018 Pore-scale visualization of methane hydrate-bearing sediments with micro-CT Geophys. Res. Lett. 45 5417-26

[8] Tang C S, Zhu C, Leng T, Shi B, Cheng Q and Zeng H 2019 Three-dimensional characterization of desiccation cracking behavior of compacted clayey soil using x-ray computed tomography Eng. Geol. 255 1-10

[9] Tomioka S, Kozaki T, Takamatsu H, Noda N, Nisiyama S, Kozai N, Suzuki S and Sato S 2010 Analysis of microstructural images of dry and water-saturated compacted bentonite samples observed with x-ray micro CT Appl. Clay Sci. 47 65-71

[10] Wang Y, Li C H and Hu Y Z 2018 Use of x-ray computed tomography to investigate the effect of rock blocks on meso-structural changes in soil-rock mixture under triaxial deformation Constr. Build. Mater. 164 386-99

[11] Abel R L, Parfitt S, Ashton N, Lewis S G, Scott B and Stringer C 2011 Digital preservation and dissemination of ancient lithic technology with modern micro-CT Comput. Graph. 35 878-84

[12] Albertin F, Bettuzzi M, Brancaccio R, Morigi M P and Casali F 2019 X-ray computed tomography in situ: an opportunity for museums and restoration laboratories Heritage 2 2028-38

[13] Keklikoglou K, Faulwetter S, Chatzinikolaou E, Wils P, Brecko J, Kvaček J, Metscher B and Arvanitidis C 2019 Micro-computed tomography for natural history specimens: a handbook of best practice protocols Eur. J. Taxon. 522

[14] Morigi M P, Casali F, Bettuzzi M, Brancaccio R and D'Errico V 2010 Application of x-ray computed tomography to cultural heritage diagnostics Appl. Phys. A 100 653-61

[15] Wilson P, Williams M A, Warnett J M, Attridge A, Ketchum H, Hay J and Smith M P 2017 Utilizing x-ray computed tomography for heritage conservation: the case of megalosaurus bucklandii 2017 IEEE Int. Instrumentation and Measurement Technology Conf. (I2MTC) pp 1-5

[16] Buffiere J Y, Maire E, Adrien J, Masse J P and Boller E 2010 In situ experiments with $\mathrm{X}$ ray tomography: an attractive tool for experimental mechanics Exp. Mech. 50 289-305 
[17] Garcea S C, Wang Y and Withers P J 2018 X-ray computed tomography of polymer composites Compos. Sci. Technol. $156305-19$

[18] Maire E and Withers P J 2014 Quantitative x-ray tomography Int. Mater. Rev. 59 1-43

[19] Salvo L et al 2003 X-ray micro-tomography an attractive characterisation technique in materials science $\mathrm{Nucl}$. Instrum. Methods Phys. Res. B 200 273-86

[20] Wiebicke M, Andò E, Herle I and Viggiani G 2017 On the metrology of interparticle contacts in sand from x-ray tomography images Meas. Sci. Technol. 28124007

[21] Rawson S D, Maksimcuka J, Withers P J and Cartmell S H 2020 X-ray computed tomography in life sciences $B M C$ Biol. 1821

[22] du Plessis A, Yadroitsava I and Yadroitsev I 2020 Effects of defects on mechanical properties in metal additive manufacturing: a review focusing on $\mathrm{x}$-ray tomography insights Mater. Des. 187108385

[23] Ontiveros S, Yagüe-Fabra J A, Jiménez R, Tosello G, Gasparin S, Pierobon A, Carmignato S and Hansen H N 2012 Dimensional measurement of micro-moulded parts by computed tomography Meas. Sci. Technol. 23125401

[24] Thompson A, Maskery I and Leach R K 2016 X-ray computed tomography for additive manufacturing: a review Meas. Sci. Technol. 27072001

[25] Villarraga-Gómez H, Herazo E L and Smith S T 2019 X-ray computed tomography: from medical imaging to dimensional metrology Prec. Eng. 60 544-69

[26] Villarraga-Gómez H, Thousand J D and Smith S T 2020 Empirical approaches to uncertainty analysis of $\mathrm{x}$-ray computed tomography measurements: a review with examples Prec. Eng. 64 249-68

[27] Withers P J et al 2021 X-ray computed tomography Nat. Rev. Methods Primers 118

[28] Shearing P, Turner M, Sinclair I, Lee P, Ahmed F, Quinn P, Leach R K, Sun W and Warnett J M 2018 EPSRC X-ray tomography roadmap 2018

[29] Dewanckele J, Boone M A, Coppens F, Van Loo D and Merkle A P 2020 Innovations in laboratory-based dynamic micro-CT to accelerate in situ research J. Microsc. 277 197-209

[30] Martz H E, Azevedo S G, Brase J M, Waltjen K E and Schneberk D J 1990 Computed tomography systems and their industrial applications Int. J. Radiat. Appl. Instrum. A 41 943-61

[31] Bultreys T, Boone M A, Boone M N, De Schryver T, Masschaele B, Van Hoorebeke L and Cnudde V 2016 Fast laboratory-based micro-computed tomography for pore-scale research: illustrative experiments and perspectives on the future Adv. Water Resour. 95 341-51

[32] BSI BS EN ISO 15708-2-2019 2019 Non-Destructive Testing-Radiation Methods for Computed Tomography Part 2: Principles, Equipment and Samples (British Standards Institution)

[33] VDI/VDE 2630 Part 1.2 2010 Computed Tomography in Dimensional Measurement_Influencing Variables on Measurement Results and Recommendations for Computed Tomography Dimensional Measurements (Institute for Innovation and Technology)

[34] Angel J and De Chiffre L 2014 Comparison on computed tomography using industrial items CIRP Ann. 63 473-6

[35] Townsend A et al 2018 An interlaboratory comparison of $\mathrm{x}$-ray computed tomography measurement for texture and dimensional characterisation of additively manufactured parts Addit. Manuf. 23 422-32

[36] Bartscher M, Sato O, Härtig F and Neuschaefer-Rube U 2014 Current state of standardization in the field of dimensional computed tomography Meas. Sci. Technol. 25064013
[37] Kiekens K, Welkenhuyzen F, Tan Y, Bleys P, Voet A, Kruth J and Dewulf W 2011 A test object with parallel grooves for calibration and accuracy assessment of industrial computed tomography (CT) metrology Meas. Sci. Technol. 22115502

[38] Léonard F, Brown S B, Withers P J, Mummery P M and McCarthy M B 2014 A new method of performance verification for $\mathrm{X}$-ray computed tomography measurements Meas. Sci. Technol. 25065401

[39] Schmitt R and Niggemann C 2010 Uncertainty in measurement for $\mathrm{x}$-ray-computed tomography using calibrated work pieces Meas. Sci. Technol. 21054008

[40] Carmignato S 2012 Accuracy of industrial computed tomography measurements: experimental results from an international comparison CIRP Ann. 61 491-4

[41] Moroni G and Petrò S 2018 A discussion on performance verification of $3 \mathrm{D} \mathrm{x}$-ray computed tomography systems Proc. CIRP 75 125-30

[42] du Plessis A et al 2019 Laboratory x-ray tomography for metal additive manufacturing: round robin test Addit. Manuf. 30100837

[43] Rodríguez-Sánchez A, Thompson A, Körner L, Brierley N and Leach R 2020 Review of the influence of noise in x-ray computed tomography measurement uncertainty Precis. Eng. 66 382-91

[44] du Plessis A, Tshibalanganda M and le Roux S G 2020 Not all scans are equal: $\mathrm{x}$-ray tomography image quality evaluation Mater. Today Commun. 22100792

[45] Bauza M B et al 2018 Realization of Industry 4.0 with high speed CT in high volume production CIRP J. Manuf. Sci. Technol. 22 121-5

[46] Hipsley C A, Aguilar R, Black J R and Hocknull S A 2020 High-throughput microCT scanning of small specimens: preparation, packing, parameters and post-processing Sci. Rep. 1019956

[47] ZEISS Xradia Context microCT 2021 Your 3D x-ray system for today with assurance for tomorrow (available at: www.zeiss.com/microscopy/int/products/x-ray-micro scopy/zeiss-xradia-context-microct.html\#downloads)

[48] Eberhorn M, Oeckl S, Stocker T and Brunner F 2016 Novel handling concept for production-integrated computed tomography 19th Conf. on Non-Destructive Testing 2016 (WCNDT)

[49] De Schryver T et al 2016 In-line NDT with x-ray CT combining sample rotation and translation $N D T \& E$ Int. 84 89-98

[50] Brunke O, Lübbehüsen J, Hansen F and Butz F F 2013 A new concept for high-speed atline and inlineCT for up to $100 \%$ mass production process control 16th Int. Congress of Metrology p 06003

[51] Warnett J M, Titarenko V, Kiraci E, Attridge A, Lionheart W R, Withers P J and Williams M A 2016 Towards in-process x-ray CT for dimensional metrology Meas. Sci. Technol. 27035401

[52] Buurlage J W, Kohr H, Jan Palenstijn W and Joost Batenburg K 2018 Real-time quasi-3D tomographic reconstruction Meas. Sci. Technol. 29064005

[53] Janssens E, De Beenhouwer J, Van Dael M, De Schryver T, Van Hoorebeke L, Verboven P, Nicolai B and Sijbers J 2018 Neural network Hilbert transform based filtered backprojection for fast inline x-ray inspection Meas. Sci. Technol. 29034012

[54] Körner L, Lawes S, Bate D, Newton L, Senin N and Leach R 2019 Increasing throughput in $\mathrm{x}$-ray computed tomography measurement of surface topography using sinogram interpolation Meas. Sci. Technol. 30125002

[55] Gajjar P, Jørgensen J S, Godinho J R A, Johnson C G, Ramsey A and Withers P J 2018 New software protocols 
for enabling laboratory based temporal CT Rev. Sci. Instrum. 89093702

[56] Finegan D P et al 2016 Quantifying bulk electrode strain and material displacement within lithium batteries via high-speed operando tomography and digital volume correlation Adv. Sci. 31500332

[57] Van Offenwert S, Cnudde V and Bultreys T 2019 Pore-scale visualization and quantification of transient solute transport using fast microcomputed tomography Water Resour. Res. 55 9279-91

[58] Beckmann F, Grupp R, Haibel A, Huppmann M, Noethe M, Pyzalla A, Reimers W, Schreyer A and Zettler R 2007 In-situ synchrotron $\mathrm{x}$-ray microtomography studies of microstructure and damage evolution in engineering materials Adv. Eng. Mater. $9939-50$

[59] Breunig T, Stock S and Brown R 1993 Simple load frame for in situ computed tomography and $\mathrm{x}$-ray tomographic microscopy Mater. Eval. 51 596-600

[60] Burch S and Lawrence P 1992 Recent advances in computerised $\mathrm{x}$-ray tomography using real-time radiography equipment $\mathrm{Br}$. J. Non-Destr. Test. 34 129-33

[61] Drake S 1993 Improved real-time x-ray technology widens the horizons of industrial computer tomography $\mathrm{Br}$. J. Non-Destr. Test. 35 580-3

[62] Bay B K, Smith T S, Fyhrie D P and Saad M 1999 Digital volume correlation: three-dimensional strain mapping using x-ray tomography Exp. Mech. 39 217-26

[63] Buljac A, Jailin C, Mendoza A, Neggers J, Taillandier-Thomas T, Bouterf A, Smaniotto B, Hild F and Roux S 2018 Digital volume correlation: review of progress and challenges Exp. Mech. 58 661-708

[64] Dall'Ara E, Peña-Fernández M, Palanca M, Giorgi M, Cristofolini L and Tozzi G 2017 Precision of digital volume correlation approaches for strain analysis in bone imaged with micro-computed tomography at different dimensional levels Front. Mater. 431

[65] Gillard F, Boardman R, Mavrogordato M, Hollis D, Sinclair I, Pierron F and Browne M 2014 The application of digital volume correlation (DVC) to study the microstructural behaviour of trabecular bone during compression J. Mech. Behav. Biomed. Mater. 29 480-99

[66] Forsberg F and Siviour C R 2009 3D deformation and strain analysis in compacted sugar using $\mathrm{x}$-ray microtomography and digital volume correlation Meas. Sci. Technol. 20095703

[67] Lachambre J, Réthoré J, Weck A and Buffiere J Y 2015 Extraction of stress intensity factors for 3D small fatigue cracks using digital volume correlation and $\mathrm{x}$-ray tomography Int. J. Fatigue 71 3-10

[68] McDonald S A and Withers P J 2014 Combining x-ray microtomography and three-dimensional digital volume correlation to track microstructure evolution during sintering of copper powder J. Strain Anal. Eng. Des. 49 257-69

[69] Blunk S, Malik A H, De Heer M I, Ekblad T, Fredlund K, Mooney S J and Sturrock C J 2017 Quantification of differences in germination behaviour of pelleted and coated sugar beet seeds using x-ray computed tomography (x-ray CT) Biomed. Phys. Eng. Express 3044001

[70] Lowe T, Garwood R J, Simonsen T J, Bradley R S and Withers P J 2013 Metamorphosis revealed: time-lapse three-dimensional imaging inside a living chrysalis $J . R$ Soc. Interface $\mathbf{1 0} 20130304$

[71] Keyes S, Gillard F, Soper N, Mavrogordato M, Sinclair I and Roose T 2016 Mapping soil deformation around plant roots using in vivo $4 \mathrm{D} \mathrm{x}$-ray computed tomography and digital volume correlation J. Biomech. 49 1802-11
[72] Bas H K, Jin W, Gupta N and Behera R K 2018 In-situ micro-CT characterization of mechanical properties and failure mechanism of cementitious syntactic foams $\mathrm{Cem}$. Concr. Compos. 90 50-60

[73] Wan K, Li G, Wang S and Pang C 2017 3D full field study of drying shrinkage of foam concrete Cem. Concr. Compos. 82 217-26

[74] Yu B, Blanc R, Soutis C and Withers P J 2016 Evolution of damage during the fatigue of $3 \mathrm{D}$ woven glass-fibre reinforced composites subjected to tension-tension loading observed by time-lapse $\mathrm{x}$-ray tomography Composites A 82 279-90

[75] Bradley R S et al 2017 Time-lapse lab-based x-ray nano-CT study of corrosion damage J. Microsc. 267 98-106

[76] Vavř́k D, Jakøubek J, Kumpova I and Pichotka M 2017 Laboratory based study of dynamical processes by 4D $\mathrm{x}$-ray CT with sub-second temporal resolution J. Instrum. $12 \mathrm{C} 02010$

[77] Ahmed S, Klassen T N, Keyes S, Daly M, Jones D L, Mavrogordato M, Sinclair I and Roose T 2016 Imaging the interaction of roots and phosphate fertiliser granules using 4D x-ray tomography Plant Soil 401 125-34

[78] Yang L, Zhang Y, Liu Z, Zhao P and Liu C 2015 In-situ tracking of water transport in cement paste using $\mathrm{X}$-ray computed tomography combined with $\mathrm{CsCl}$ enhancing Mater. Lett. 160 381-3

[79] Feldkamp L, Davis L C and Kress J 1984 Practical cone-beam algorithm J. Opt. Soc. Am 1 612-9

[80] Aloisi V, Schlecht J, Ferley E and Carmignato S 2019 Characterization of the effects of detector angular misalignments and accuracy enhancement of x-ray CT dimensional measurements Conf. on Industrial Computed Tomography (Padova, Italy)

[81] Dewulf W, Ferrucci M, Ametova E, Heřmánek P, Probst G, Boeckmans B, Craeghs T and Carmignato S 2018 Enhanced dimensional measurement by fast determination and compensation of geometrical misalignments of x-ray computed tomography instruments CIRP Ann. 67 523-6

[82] Ferrucci M, Leach R K, Giusca C, Carmignato S and Dewulf W 2015 Towards geometrical calibration of x-ray computed tomography systems-a review Meas. Sci. Technol. 26092003

[83] Lüthi M, Bircher B A, Meli F, Küng A and Thalmann R 2019 $\mathrm{X}$-ray flat-panel detector geometry correction to improve dimensional computed tomography measurements Meas. Sci. Technol. 31035002

[84] Carmignato S, Aloisi V, Medeossi F, Zanini F and Savio E 2017 Influence of surface roughness on computed tomography dimensional measurements CIRP Ann. 66 499-502

[85] Lifton J J, Malcolm A A and McBride J W 2015 On the uncertainty of surface determination in X-ray computed tomography for dimensional metrology Meas. Sci. Technol. 26035003

[86] Hiller J, Maisl M and Reindl L M 2012 Physical characterization and performance evaluation of an x-ray micro-computed tomography system for dimensional metrology applications Meas. Sci. Technol. 23085404

[87] Villarraga-Gómez H, Thousand J D, Morse E P and Smith S 2015 CT measurements and their estimated uncertainty: the significance of temperature and bias determination Proc. 15th Int. Conf. on Metrology and Properties of Engineering Surfaces

[88] Lifton J, Malcolm A and McBride J 2015 An experimental study on the influence of scatter and beam hardening in x-ray CT for dimensional metrology Meas. Sci. Technol. 27015007

[89] Bartscher M, Staude A, Ehrig K and Ramsey A 2012 The influence of data filtering on dimensional measurements 
with CT Conf. on Non Destructive Testing (WCNDT) (Durban, South Africa)

[90] Stolfi A, Thompson M K, Carli L and De Chiffre L 2016 Quantifying the contribution of post-processing in computed tomography measurement uncertainty Proc. CIRP 43 297-302

[91] Villarraga-Gómez H, Lee C and Smith S T 2018 Dimensional metrology with X-ray CT: a comparison with CMM measurements on internal features and compliant structures Precis. Eng. 51 291-307

[92] Buratti A, Grozmani N, Voigtmann C, Sartori L V and Schmitt R H 2018 Determination of the optimal imaging parameters in industrial computed tomography for dimensional measurements on monomaterial workpieces Meas. Sci. Technol. 2913

[93] Chahid M, Townsend A, Liu A, Bills P, Sperling P and Racasan R 2020 Optimizing X-ray computed tomography settings for dimensional metrology using 2D image analysis 4th Symp. on Structural Integrity of Additive Manufactured Materials and Parts (ASTM Int.) pp 88-101

[94] BSI BS EN ISO 19232-5-2018 2018 Non-Destructive Testing_Image Quality of Radiographs, Determination of the Image Unsharpness and Basic Spatial Resolution Value using Duplex Wire-Type Image Quality Indicators (British Standards Institution)

[95] Stolfi A, De Chiffre L and Kasperl S 2018 Error sources Industrial X-Ray Computed Tomography (Cham: Springer International Publishing) pp 143-84

[96] du Plessis A, Broeckhoven C, Guelpa A and le Roux S G 2017 Laboratory x-ray micro-computed tomography: a user guideline for biological samples GigaScience 6 1-11

[97] BSI BS EN 12543-1:1999 1999 Non-Destructive Testing Characteristics of Focal Spots in Industrial X-Ray Systems for Use in Non-Destructive Testing Scanning Method (British Standards Institution)

[98] Kueh A, Warnett J M, Gibbons G J, Brettschneider J, Nichols T E, Williams M A and Kendall W S 2016 Modelling the penumbra in computed tomography 1 J. X-Ray Sci. Technol. 24 583-97

[99] Molteni R 2020 X-ray imaging: fundamentals of x-ray Micro-Computed Tomography (Micro-CT) in Medicine and Engineering (Berlin: Springer International Publishing) ch 2, pp 7-25

[100] Punnoose J, Xu J, Sisniega A, Zbijewski W and Siewerdsen J H 2016 Technical note: spektr 3.0 A computational tool for $\mathrm{x}$-ray spectrum modeling and analysis Med. Phys. 43 4711-7

[101] Kruth J P, Bartscher M, Carmignato S, Schmitt R, De Chiffre L and Weckenmann A 2011 Computed tomography for dimensional metrology CIRP Ann. 60 821-42

[102] Herman G T 2009 Fundamentals of computerized tomography: image reconstruction from projections $A d v$. Pattern Recognit. (London: Springer Science \& Business Media) (https://doi.org/10.1007/978-1-84628-723-7)

[103] Villarraga-Gómez H and Smith S T 2020 Effect of the number of projections on dimensional measurements with X-ray computed tomography Precis. Eng. 66 445-56

[104] Luksic S A, Pokorny R, George J, Hrma P, Varga T, Reno L R, Buchko A C and Kruger A A 2020 In situ characterization of foam morphology during melting of simulated waste glass using $\mathrm{x}$-ray computed tomography Ceram. Int. 46 17176-85

[105] Luksic S A, Pokorny R, Hrma P, Varga T, Rivers E L, Buchko A C, Klouzek J and Kruger A A 2021 Through a glass darkly: in-situ X-ray computed tomography imaging of feed melting in continuously fed laboratory-scale glass melter Ceram. Int. 47 15807-18
[106] Bultreys T, Boone M A, Boone M N, De Schryver T, Masschaele B, Van Loo D, Van Hoorebeke L and Cnudde V 2015 Real-time visualization of Haines jumps in sandstone with laboratory-based microcomputed tomography Water Resour. Res. 51 8668-76

[107] Van Offenwert S, Cnudde V, Boone M and Bultreys T 2021 Fast micro-computed tomography data of solute transport in porous media with different heterogeneity levels Sci. Data 818

[108] Eggert A, Müller M, Nachtrab F, Dombrowski J, Rack A and Zabler S 2014 High-speed in-situ tomography of liquid protein foams Int. J. Mater. Res. 105 632-9

[109] Yan D, Zhang Z, Luo Q and Yang X 2017 A novel mouse segmentation method based on dynamic contrast enhanced micro-CT images PLoS One $12 \mathrm{e} 0169424$

[110] Bubba T A, Heikkilä T, Help H, Huotari S, Salmon Y and Siltanen S 2020 Sparse dynamic tomography: a shearlet-based approach for iodine perfusion in plant stems Inverse Problems 36094002

[111] Heyndrickx M, Bultreys T, Goethals W, Van Hoorebeke L and Boone M N 2020 Improving image quality in fast, time-resolved micro-CT by weighted back projection $S c i$. Rep. 1018029

[112] Thompson W M, Lionheart W R, Morton E J, Cunningham M and Luggar R D 2015 High speed imaging of dynamic processes with a switched source $\mathrm{x}$-ray CT system Meas. Sci. Technol. 26055401

[113] Myers G R, Kingston A M, Varslot T K, Turner M L and Sheppard A P 2011 Dynamic tomography with a priori information Appl. Opt. 50 3685-90

[114] Sheppard A et al 2014 Techniques in helical scanning, dynamic imaging and image segmentation for improved quantitative analysis with x-ray micro-CT Nucl. Instrum. Methods Phys. Res. B 324 49-56

[115] Heyndrickx M, Boone M, De Schryver T, Bultreys T, Goethals W, Verstraete G, Vanhoorne V and Van Hoorebeke L 2018 Piecewise linear fitting in dynamic micro-CT Mater. Charact. 139 259-68

[116] Myers G R, Geleta M, Kingston A M, Recur B and Sheppard A P 2015 Bayesian approach to time-resolved tomography Opt. Express 23 20062-74

[117] Fischer A, Lasser T, Schrapp M, Stephan J and Noël P B 2016 Object specific trajectory optimization for industrial x-ray computed tomography Sci. Rep. 619135

[118] Gruse J N et al 2020 Application of compact laser-driven accelerator x-ray sources for industrial imaging $\mathrm{Nucl}$. Instrum. Methods Phys. Res. A 983164369

[119] Hornberger B, Kasahara J, Gifford M, Ruth R and Loewen R 2019 A compact light source providing high-flux, quasi-monochromatic, tunable x-rays in the laboratory Proc. SPIE 111101111003

[120] Jørgensen J S and Sidky E Y 2015 How little data is enough? Phase-diagram analysis of sparsity-regularized x-ray computed tomography Phil. Trans. R. Soc. A 37320140387

[121] Jørgensen J S, Coban S B, Lionheart W R and Withers P J 2016 Effect of sparsity and exposure on total variation regularized x-ray tomography from few projections 4 th Int. Conf. on Image Formation in X-Ray Computed Tomography pp 279-82

[122] Coban S B, Lionheart W R B and Withers J P 2021 Assessing the efficacy of tomographic reconstruction methods through physical quantification techniques Meas. Sci. Technol. 32075404

[123] Coban S B, Withers P, Lionheart W and McDonald S A 2015 When do the iterative reconstruction methods become worth the effort? 13th Int. Meeting on Fully Three-Dimensional Image Reconstruction in Radiology and Nuclear Medicine 
[124] Biguri A, Dosanjh M, Hancock S and Soleimani M 2016 TIGRE: a MATLAB-GPU toolbox for CBCT image reconstruction Biomed. Phys. Eng. Express 2055010

[125] Jørgensen J S et al 2021 Core imaging library-part I: a versatile Python framework for tomographic imaging (arXiv:2102.04560)

[126] Van Aarle W, Palenstijn W J, De Beenhouwer J, Altantzis T, Bals S, Batenburg K J and Sijbers J 2015 The ASTRA toolbox: a platform for advanced algorithm development in electron tomography Ultramicroscopy $15735-47$

[127] Wadeson N and Basham M 2016 Savu: a python-based, MPI framework for simultaneous processing of multiple, $\mathrm{N}$-dimensional, large tomography datasets (arXiv:1610.08015)

[128] Pelt D M, Batenburg K J and Sethian J A 2018 Improving tomographic reconstruction from limited data using mixed-scale dense convolutional neural networks J. Imaging 4128 